\title{
Statistics of Shrinkage Test Data
}

REFERENCE: Wittmann, F. H., Bažant, Z. P., Alou, F., and Kim, J.-K., "Statiatics of Shrinkage Test Data," Cement, Concrete, and Aggregates. CCAGDP, Vol. 9, No. 2, Winter 1987, pp. 129-153.

ABSTRACT: A large series of concrete shrinkage tests which can be used for statistical purposes is reported. The series involves two groups of 36 identical cylindrical specimens, with a diameter of $83 \mathrm{~mm}$ for Group 1 and $160 \mathrm{~mm}$ for Group 2. Statistical analysis of shrinkage strains and strain rates is presented, and the goodness of fit by normal distribution, log-normal distribution, and gamma distribution is analyzed. Correlations between the values at various times are determined.

The study reveals only the intrinsic randomness of the shrinkage process (along with measurement errors) and omits the superimposed uncertainties due to randomness of environment and to prediction uncertainties in the influence of material composition, curing, and other factors. It is found that the coefficient of variation of shrinkage values first decreases with time and then levels off at 6 to $9 \%$. The normal. lognormal, and gamma distributions all give acceptable fits of the ob. served distributions. There are small systematic deviations from normal distribution: the distributions are slightly skewed to the right and slightly platykurtic. The skewness is more pronounced for the distribution of shrinkage rates, and asymmetric distributions such as gamma give slightly better fits. The correlation of single specimen shrinkage deviations from the group mean at various times is characterized by correlation coefficients. Their value is found to decrease slowly as the length of the time interval increases. For shrinkage rates, this decrease is much faster. The standard deviation of the single specimen shrinkage from its smoothed shrinkage curve is much less than (about one third of) the standard deviation of the single specimen relative to the mean for all specimens. The standard deviation of all single-specimen smoothed shrinkage curves at one time is almost the same as (about $95 \%$ of) the standard deviation of the original unsmoothed data for that time.

KEYWORDS: concrete, cements, shrinkage, drying, tests, measurements, statistics, probabilistic analysis, random errors, shrinkage prediction

Predictions of shrinkage of concrete have a large statistical variability, which is caused: (1) by the uncertainty in the influence of the composition and curing history of concrete; $(2)$ by the random variability of environment; and (3) by intrinsic randomness of concrete due to the random nature of the shrinkage mechanism itself and to our uncertainty in the knowledge of the law governing

'Professor, Department of Materials, Swiss Federal Institute of Technology, Lausanne, Switzerland.

${ }^{2}$ Professor, Department of Civil Engineering, Northwestern University, Evanston, IL 60208.

${ }^{3}$ Head, Concrete Technology and Material Testing Section, Department of Materials, Swiss Federal Institute of Technology, Lausanne, Switzerland.

${ }^{4}$ Assistant professor, Department of Civil Engineering, Korea Advanced Institute of Science and Technology, Seoul, Korea; formerly graduate research assistant, Northwestern University.

(C) 1987 by the American Society for Testing and Materials shrinkage. In structural design, each of these three sources of randomness should be treated separately $[1-6]$.

The purpose of the present study is to generate statistical data for the third source of randomness, the intrinsic uncertainty. The experimental information on this type of uncertainty $[7-10]$ is rather limited, and no sufficiently large series of test data which would permit extracting reliable statistics seems to exist. Therefore, a large series of concrete shrinkage tests has been carried out in Lausanne under a cooperative project between the Department of Materials of the Swiss Federal Institute of Technology and the Center for Concrete and Geomaterials at Northwestern University. The purpose of this paper is to report the test results and present their statistical analysis, whose concept was developed in Bažant's report $[11]$. Two preceding papers $[12.13]$ used the same data to study the problems of extrapolation of short-time shrinkage data by regression and by Bayesian analysis.

\section{Description of Tests}

The experimental study involved one series of 35 cylindrical specimens of diameter $160 \mathrm{~mm}$ and another series of 36 cylindrical specimens of diameter $83 \mathrm{~mm}$ (Fig. 1). (The effect of specimen size, however, is not analyzed here since this has been done in preceding papers $[12,13]$.) The length of each cylinder was double its diameter. The mean standard cylindrical 28-day strength of the concrete was $f_{c}^{\prime}=33.2 \mathrm{MPa}(4814 \mathrm{psi})$ and its modulus of elasticity (according to DIN 1045) at 28 days was $36.3 \mathrm{kN} / \mathrm{mm}^{2}$. No admixtures, plasticizers, or air-entraining agents were used. The specific mass of concrete was $2418 \mathrm{~kg} / \mathrm{m}^{3}$, and $1 \mathrm{~m}^{3}$ contained $350 \mathrm{~kg}$ of cement, $168 \mathrm{~kg}$ of water, $608 \mathrm{~kg}$ of fine sand from 0 to $4 \mathrm{~mm}$ size, $399 \mathrm{~kg}$ of coarse sand from 4 to $8 \mathrm{~mm}$ size, $399 \mathrm{~kg}$ of fine gravel from 8 to $16 \mathrm{~mm}$ size, and $494 \mathrm{~kg}$ of coarse gravel from 16 to $31.5 \mathrm{~mm}$ size. The corresponding volume fractions were 0.113 , $0.168,0.226,0.148,0.148$, and 0.184 , respectively, plus 0.013 for air. The cement (of type CPN from the plant at Eclepens near Lausanne) was approximately of ASTM Type I, with fineness defined by surface area $2900 \mathrm{~m}^{2} / \mathrm{g}$ (Blaine). All aggregate was from a glacial moraine, of rounded shapes. Mineralogically, all aggregate was composed of 40 to $46 \%$ calcite, 29 to $32 \%$ quartz, 8 to $13 \%$ residue of crystalline rocks of multimineral composition, predominantly quartzitic, and 12 to $18 \%$ of composite grains, essentially quartzitic. All the specimens were cast from one batch of concrete. The specimens were cured in a sealed state and were kept sealed in their molds (made of PVC) until the instant of exposure to the drying environment. This type of curing is preferable to curing in water bath. which has recently been found to cause nonuniform water intake, significant swelling stresses, and microcracking. The envi- 


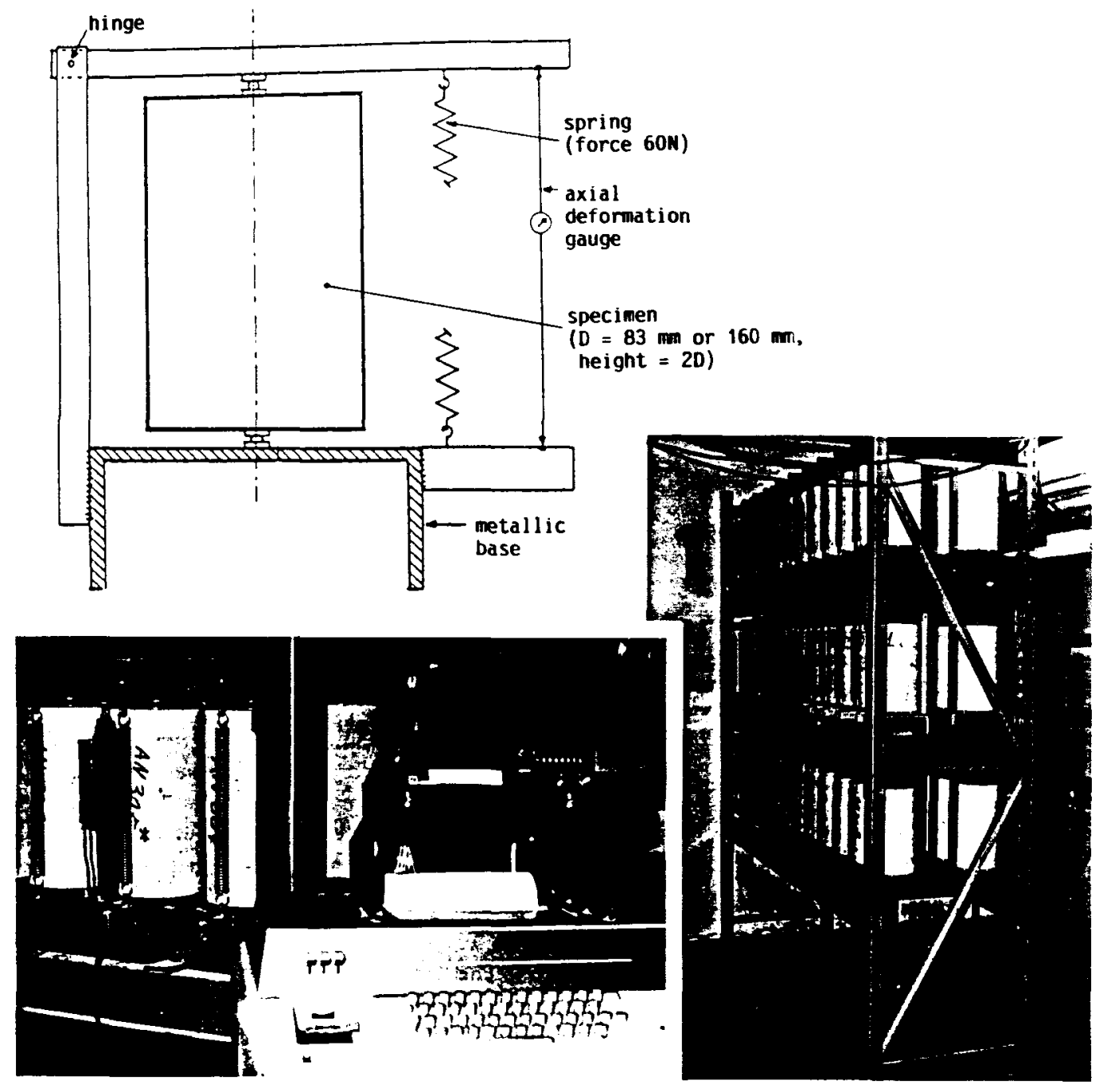

FIG. 1-Photographs of test specimens and laboratory setup.

ronmental humidity was $65 \pm 5 \%$, and the room temperature was $18 \pm 1^{\circ} \mathrm{C}$ throughout the casting, curing, and shrinkage.

The shrinkage deformations were measured by taking length readings between the ends of the specimens along the cylinder axis. The deformations of all specimens were measured with one dial gauge which had contacts made of steel balls that fit into ringshaped steel targets glued to the specimens. To be able to record the very initial shrinkage, the targets for deformation measurements had been attached before the molds were stripped. All specimens were exposed to drying at the age of seven days and shrinkage measurements started immediately. The initial reading, representing the zero strain for Tables 1 and 2, was taken within 1 min after the stripping of the mold (it is important to take the first reading promptly; in previous tests the first reading was apparently taken much later, causing a significant initial shrinkage to go unrecorded).

\section{Statistics for the Group of Specimens and Their Evolution with Time}

The shrinkage strains $\epsilon_{\text {sh }}$ measured for specimens $i=1,2, \ldots$, $N$ are reported in Table 1 (for diameter $83 \mathrm{~mm}, N=36$ ) and Ta- ble 2 (for $160 \mathrm{~mm}, N=35$ ) in terms of their mean $\bar{\epsilon}_{r}$ at time $t_{r}(r=$ $1,2,3, \ldots, n)$ and the deviations from the mean, $\epsilon_{i, r}-\bar{\epsilon}_{r}$, where $\epsilon_{i, r}$ is the shrinkage strain $\epsilon_{s h}$ of specimen number $i$ at time $t_{r}$. Time

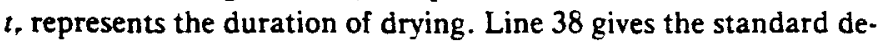
viation $s_{r}$ of the set of all $\epsilon_{i, r}$-values at time $t_{r} ; s_{r}=\Sigma_{i}\left[\left(\epsilon_{i, r}-\bar{\epsilon}_{r}\right)^{2} /\right.$ $(N-1)]^{1 / 2}$ where $N-1$ is used instead of $N$ to obtain an unbiased estimate [14,15]. Line 39 gives the coefficient of variation $\omega_{r}=$ $s_{r} / \bar{\epsilon}_{r}$ of the values $\epsilon_{i, r}$. Considering that in theory an infinite number of samples could be cast and that the available $N$ specimens represent a sample drawn from this infinite population, the standard deviation and the coefficient of variation of the mean value $\bar{\epsilon}_{\text {, }}$ are $s_{r} / \sqrt{N}$ and $\omega_{r} / \sqrt{N}[14-16](\sqrt{N}=6$ or 5.92).

Lines 40-43 give the mean $\bar{\epsilon}_{r}^{L}$ of the values of $\log \epsilon_{i, r}$, the values of $10^{i_{r}}$, the standard deviation $s_{r}^{L}$ of these values, and their coefficient of variation $\omega_{r}^{L}$. These characteristics may be used for determining the log-normal distribution of shrinkage strains, which has the advantage that it does not admit negative shrinkage strain values which are physically impossible (if measurement ertor is eliminated).

Another distribution which does not permit negative strain values and is in a certain sense better justified physically [17] is the gamma distribution of strain. It has the probability density $f(\epsilon)=$ 
$\beta^{-\alpha} \epsilon^{\alpha-1} e^{-\epsilon / \beta} / \Gamma(\alpha)$ for $\epsilon>0$ (and 0 for $\epsilon<0$ ), where $\Gamma(\alpha)$ is the gamma function, that is, $\Gamma(\alpha)=\int_{0}^{\infty} \epsilon^{\alpha-1} e^{-\epsilon} d \epsilon, \alpha$ is the scale parameter and $\beta$ is the shape parameter $(\alpha>0, \beta>0)$. As is well known [18], the mean and standard deviation of the gamma distribution are $\bar{\epsilon}=\alpha \beta$ and $s=\beta \sqrt{\alpha}$. From this it follows that $\alpha=\left(\bar{\epsilon}_{r}\right)$ $\left.s_{r}\right)^{2}, \beta=s_{r}^{2} / \bar{\epsilon}_{r} ;$ these parameters are evaluated for the present data on Lines 44 and 45.

As measures of the deviations from the normal distributions of $\epsilon_{i, r}$, lines 46 and 47 list the skewness, $m$, and the kurtosis, $k$, defined for time $t_{r}$ as in Ref 14, p. 62

$$
\begin{aligned}
& m=\frac{1}{s_{r}^{3}}\left[\frac{1}{N} \sum_{i=1}^{N}\left(\epsilon_{i, r}-\bar{\epsilon}_{r}\right)^{3}\right] \text { and } \\
& k=\frac{1}{s_{r}^{4}}\left[\frac{1}{N} \sum_{i=1}^{N}\left(\epsilon_{i, r}-\bar{\epsilon}_{r}\right)^{4}\right]
\end{aligned}
$$

Note that for normal distributions $m=0$ and $k=3$. When $k>3$, the distribution is leptokurtic (that is, it has a sharp peak and long tails), and for $k<3$, the distribution is platykurtic (that is, it has a flat peak and short tails, a shape reminding of the platypus). Lines 48 and 49 give the skewness $m^{L}$ and kurtosis $k^{L}$ for the distribution of $\log \epsilon_{i, r}$, which indicates deviations from the log-normal distribution of shrinkage strain.

To evaluate the goodness of fit by various distributions, Figs. 2-3 show for the two groups of $N$ specimens $(N=36$ or 35$)$ the cumulative frequency distributions of the $\epsilon_{i, r}$ values. Among the various types of cumulative frequency distribution plots we chose the plot introduced by Blom and Kimball $[16,19,20]$, which was proven to be optimum. For this plot, the plotting positions are $(i-$ $0.375) /(N+0.25)$, which is superior to either $(i-0.5) / N$ or $i /$ $(N+1)$ as customarily used ( $i$ is the number of data values $\leq \epsilon_{i, r}$ ). Figures 2-3 show these cumulative plots for the actual shrinkage strains $\epsilon_{i, r}$ (at four different times $t_{r}=t-t_{0}$, and for both specimen diameters $D$ ). Figures 4-5 show the same for $\log \epsilon_{i, r}$. If the distributions of $\epsilon_{i, r}$ or $\log \epsilon_{i, r}$ were normal and perfect, then the plots would have to be straight lines. Thus, vertical deviations from the least-square fit by a straight line represent errors. These errors are characterized by their coefficients of variation $\omega_{Y \mid X}$ and $\omega_{Y \mid X}^{L}$, listed on lines 50-51 of Tables 1-2, pertaining to the normal and log-normal distributions, respectively.

For the modeling of shrinkage as a stochastic process with independent increments, which is theoretically superior to the modeling of shrinkage as a stochastic process with independent total shrinkage values at subsequent times [17], it is useful to know the statistics of the shrinkage increments. They may be characterized by the statistics of the shrinkage rates, which may be approximately calculated for specimen number $i$ as

$$
\left.\dot{\epsilon}_{i, r}=\frac{\epsilon_{i, r}-\epsilon_{j, r-1}}{t_{r}-t_{r-1}} \quad \text { at time } \sqrt{t_{r-1} t_{r}}\right)
$$

The rate estimated from interval $\left(t_{r-1}, t_{r}\right)$ is best referred to the center of the interval in $\log t$-scale, which is $\sqrt{t_{r-1} t_{r}}$. Lines 53-57 of the tables give for the entire group of specimens $(i=1, \ldots, N)$ the mean of the shrinkage rates $\bar{\epsilon}_{r}^{\prime}$, their standard deviation $s_{r}^{\prime}$, their coefficient of variations $\omega_{r}^{\prime}$, skewness $m_{r}^{\prime}$, and kurtosis $k_{r}^{\prime}$. Furthermore, lines 58-62 give the statistics of $\log \dot{\epsilon}_{i, r}$, that is, their mean $\overline{\log \dot{\epsilon}_{r}}$, standard deviation $\dot{s}_{r}^{\log }$, coefficient of variation $\dot{\omega}_{r}^{\log }$, skewness $\dot{m}_{r}^{\text {log }}$, and kurtosis $\dot{k}_{r}^{\text {log }}$.
Since the initial evolution of shrinkage is simpler in the log-log plot, it is also useful to consider the rates $\operatorname{of} \log \epsilon_{s h}$ in $\log t$, calculated as

$$
\epsilon_{i, r}^{\prime L}=\frac{\log \epsilon_{i, r}-\log \epsilon_{i, r-1}}{\log t_{r}-\log t_{r-1}}
$$

Their mean $\bar{\epsilon}_{r}^{\prime L}$, standard deviation $s_{r}^{\prime L}$, and coefficient of variation $\omega_{r}^{\prime L}$ are listed in lines 63-65. Furthermore, lines $66-67$ give the scale parameter $\alpha^{\prime}$ and the shape parameter $\beta^{\prime}$ for the gamma distribution of shrinkage rates $\dot{\epsilon}_{i, r}$, determined as $\alpha^{\prime}=\left(\bar{\epsilon}_{r}^{\prime} / s_{r}^{\prime}\right)^{2}$ and $\beta^{\prime}=s_{r}^{\prime 2} / \bar{\epsilon}_{r}^{\prime}$.

Figures 6-9 help to evaluate the goodness of fit of the shrinkage rates by the normal and log-normal distributions. These figures show the cumulative frequency distributions of $\dot{\epsilon}_{i, r}$ and $\epsilon_{i, r}^{\prime}$, plotted in the same manner as Figs. 2-5. If these distributions were perfect, these plots would have to be straight lines, and so the vertical deviations from the straight-line fit are a measure of the deviation from the theoretical distribution. These vertical deviations may be characterized by their coefficients of variation $\omega_{Y \mid X}^{\prime}$ and $\omega_{Y \mid X}^{\prime}$ for the normal and log-normal distributions, respectively, which are listed in lines 68-69.

The probability density distributions corresponding to the aforementioned cumulative distributions are plotted in Figs. 10-13.

\section{Statistics of the Time Series}

Aside from the statistics for each group of specimens at a fixed time, it is also of interest to determine correlation of shrinkage strains at various times for each particular specimen. This correlation may be characterized, for example, by the correlation coefficient [Ref 14, p. 74; Ref 15, p. 161] for $\epsilon_{s h}$ and $\log \epsilon_{s h}$ at two different times $t_{r}$ and $t_{q}$, which is obtained as the covariance divided by the product of standard deviations, that is,

$$
\begin{gathered}
c\left(t_{r}, t_{q}\right)=\frac{1}{s_{r} s_{q}} \frac{1}{N-1} \sum_{i=1}^{N}\left(\epsilon_{i, r}-\bar{\epsilon}_{r}\right)\left(\epsilon_{i, q}-\bar{\epsilon}_{q}\right) \\
c^{L}\left(t_{r}, t_{q}\right)=\frac{1}{s_{r}^{L} s_{q}^{L}} \frac{1}{N-1} \sum_{i=1}^{N}\left(\log \epsilon_{i, r}-\bar{\epsilon}_{r}^{L}\right)\left(\log \epsilon_{i, q}-\bar{\epsilon}_{q}^{L}\right)
\end{gathered}
$$

We use here $N-1$ instead of $N$ in the usual definition $[14,15]$ because we must require that for $t_{q}=t$, the value of $c$ be 1.0 (perfect correlation). The values of these correlation moments for all possible combinations of shrinkage times 3,21, 91, 554, and 1399 days are listed in Table 3 . A correlation coefficient equal to 1.0 means perfect correlation (functional dependence) and 0 means no correlation. The rates in this table are calculated from the intervals $(2,3),(14,21),(52,91),(412,554)$, and $(1105,1399)$ (in days). The autocorrelation function of the random process of shrinkage could be constructed from Table 3 .

The normalized correlation moments can be similarly defined for the deviations of the single specimen shrinkage rates from the group mean

$$
\begin{gathered}
c^{\prime}\left(t_{r}, t_{q}\right)=\frac{1}{s_{r}^{\prime} s_{q}^{\prime}} \frac{1}{N-1} \sum_{i=1}^{N}\left(\dot{\epsilon}_{j, r}-\bar{\epsilon}_{r}^{\prime}\right)\left(\dot{\epsilon}_{i, q}-\bar{\epsilon}_{q}^{\prime}\right) \\
c^{\prime L}\left(t_{r}, t_{q}\right)=\frac{1}{s_{r}^{\prime L} s_{q}^{\prime L}} \frac{1}{N-1} \sum_{i=1}^{N}\left(\epsilon_{i, r}^{\prime L}-\bar{\epsilon}_{r}^{\prime L}\right)\left(\epsilon_{i, q}^{\prime L}-\bar{\epsilon}_{q}^{\prime L}\right)
\end{gathered}
$$




\begin{tabular}{|c|c|c|c|c|c|c|c|c|c|c|c|c|c|c|c|c|c|c|c|c|c|}
\hline & $\begin{array}{l}\text { time } t \\
\text { in days) }\end{array}$ & .0104 & 0.042 & 0.208 & 0.375 & 1 & 2.08 & 3 & 6 & 8 & 14 & 21 & 34 & 52 & 91 & 169 & 258 & 412 & 554 & 1105 & 1399 \\
\hline 1 & & -3.5 & 5.1 & -1.0 & -4.1 & -2.8 & 0.3 & 3.3 & 5.5 & 8.1 & 15.2 & 18.0 & 26.1 & 28.6 & 18.1 & 13.1 & 26.5 & 22.7 & 29.6 & 29.9 & 32.3 \\
\hline 2 & & 3.9 & -2.3 & -8.4 & -12.6 & -13.8 & -18 & -22.4 & -26.4 & -27 & -30.2 & -29.8 & -33.9 & -41.3 & -49.3 & -67.8 & -74.0 & -79.0 & & -91.4 & \\
\hline 3 & & -2.2 & -7.2 & -9.6 & -11.4 & -8.9 & -7.0 & -7.7 & -8.0 & -10.3 & -9.3 & -10.2 & -15.6 & -24.1 & -35.8 & -49.4 & -51.9 & -53.3 & -48.8 & -44.8 & -42.5 \\
\hline 4 & & -1.0 & -2.3 & -3.5 & -5.3 & -2.8 & -2.1 & -1.6 & 1.8 & 2.0 & 5.4 & 5.7 & 5.3 & 4.1 & 1.0 & -5.3 & -4.1 & -3.0 & -5.9 & -1.9 & -3.2 \\
\hline 5 & & 2.7 & 0.2 & -3.5 & -6.5 & -5.2 & -4.6 & -2.8 & 0.6 & 0.7 & 0.5 & 0.8 & -3.3 & -9.4 & -17.4 & -24.9 & -26.2 & -27.5 & -30.4 & -22.8 & -24.1 \\
\hline 6 & & -5.9 & -12.1 & -13.3 & -9.0 & -6.5 & -2.1 & -1.6 & 4.3 & 5.7 & 15.2 & 21.7 & 23.7 & 28.6 & 24.2 & 29.0 & 40.0 & 37.4 & 46.8 & 43.4 & 53.1 \\
\hline 7 & & 7.6 & 7.5 & 7.6 & 8.2 & 10.7 & 10.1 & 9.4 & 6.7 & 4.4 & 5.4 & 5.7 & 2.8 & -0.8 & -6.4 & -12.7 & -15.1 & -16.5 & -19.4 & -15.4 & -14.3 \\
\hline 8 & & -2.2 & -3.5 & -2.2 & -2.8 & 5.8 & 16.2 & 20.5 & 27.5 & 30.2 & 44.6 & 49.8 & 59.2 & 71.5 & 78.2 & 80.5 & 95.1 & 102.4 & 106.8 & 103.5 & 108.3 \\
\hline 9) & & -3.5 & -3.5 & -5.9 & -6.5 & -1.6 & 4.0 & 9.4 & 16.5 & 17.9 & 27.4 & 30.2 & 34.7 & 39. & 35.3 & 40.0 & 48.6 & 54.6 & 55.4 & 49.5 & 59.3 \\
\hline 10 & & -2.2 & -4.7 & -12.1 & -12.6 & -13.8 & -15.6 & -16.3 & -17.8 & -18.9 & -19.1 & -22.5 & -26.6 & -32.7 & -38.3 & -51.9 & -56.8 & -60.6 & -66.0 & -64.4 & -63.3 \\
\hline 11 & & -3.5 & -4.7 & -10.8 & -13.9 & -15.0 & -14.4 & -15.1 & -19.0 & -21.3 & -24.0 & -26.1 & -31.5 & -36.4 & -37.0 & -48.2 & -51.9 & -52.0 & -52.5 & -49.7 & -52.3 \\
\hline 12 & & -2.2 & -7.2 & -13.3 & -17.5 & -21.2 & -23.0 & -21.2 & -15.4 & -16.4 & -10.6 & -6.5 & -7.0 & -6.9 & -10.1 & -13.9 & -13.9 & -16.5 & -18.2 & -16.6 & -20.4 \\
\hline 13 & & -1.0 & 0.2 & -1.0 & -1.6 & -4.0 & -5.8 & -5.3 & -0.6 & 0.7 & 1.7 & 3.3 & 6.5 & 5.3 & 9.5 & 13.1 & 17.9 & 19.0 & 18.6 & 23.8 & 20.1 \\
\hline 14 & & -2.2 & -3.5 & -4.7 & -7.7 & -12.6 & -18.1 & -20.0 & -25.2 & -31.1 & -37.5 & -40.8 & -47.4 & -59. & -68.9 & -78.8 & -91.1 & -96.2 & -97.8 & -95.1 & -97.6 \\
\hline 15 & & -10.8 & -9.6 & -7.1 & -9.0 & -4.0 & -2.1 & -1.6 & 1.8 & 3.2 & 11.5 & 18.0 & 26.1 & 37.2 & 27.9 & 56.0 & 69.4 & 80.3 & 83.5 & 87.5 & 83.8 \\
\hline 16 & & -9.6 & -6.0 & -4.7 & -5.3 & -6.5 & -5.8 & -7.7 & -11.7 & -14.0 & -16.7 & -18.8 & -22.9 & -30.2 & -35.8 & -44.5 & -54.4 & -59.4 & -63.5 & -68.1 & -64.5 \\
\hline 17) & $\varepsilon_{\text {oh }}-\bar{\varepsilon}_{\text {or }}$ & 1.4 & 0.2 & 2.7 & 2.1 & 4.6 & 5.2 & 4.5 & 3.0 & 0.7 & 1.7 & 3.3 & 4.1 & 4.1 & 4.6 & 3.3 & 3.2 & 3.1 & 2.7 & 4.2 & 1.7 \\
\hline 18 & & 3.9 & 5.1 & 3.9 & 4.5 & 9.5 & 13.8 & 14.3 & 17.7 & 20.4 & 23.8 & 27.8 & 37.1 & 43.3 & 50.0 & 58.4 & 64.5 & 73.0 & 72.5 & 69.1 & 69.1 \\
\hline 19 & & -3.5 & -3.5 & -3.5 & -5.3 & -6.5 & -9.5 & -10.2 & -15.4 & -20.1 & -22.8 & -26.1 & -37.6 & -46.2 & -54.2 & -64.1 & -75.2 & -81.5 & -90.5 & -90.2 & -91.5 \\
\hline 20 & & -9.6 & -8.4 & -8.4 & -7.7 & -5.2 & -3.4 & -2.8 & -3.1 & -4.2 & -2.0 & 0.8 & 2.8 & 9.0 & 18.1 & 26.6 & 32.6 & 39.9 & 39.4 & 43.4 & 39.7 \\
\hline 21 & & -1.0 & 1.4 & 1.4 & 4.5 & 7.0 & 8.9 & 8.2 & 10.4 & 13.0 & 20.1 & 25.3 & 31.0 & 42.1 & 48.7 & 56.0 & 59.6 & 64.4 & 63.9 & 65.5 & 66.6 \\
\hline 22 & & -2.2 & -2.3 & -4.7 & -5.3 & -7.7 & -13.2 & -16.3 & -25.2 & -31.1 & -40.0 & -47.0 & -59.7 & -73.1 & -76.3 & -92.3 & -109.5 & -115.8 & -124.8 & -128.2 & -123.3 \\
\hline 23 & & -9.6 & -6.0 & -7.1 & -7.7 & -8.9 & -9.5 & -9.0 & -0.6 & 0.7 & 6.6 & 9.4 & 9.0 & 17.6 & 34.0 & 36.4 & 38.8 & 38.6 & 38.2 & 38.5 & 36.0 \\
\hline 24 & & -5.9 & -1.1 & -5.9 & -6.5 & -15.0 & -19.3 & -18.8 & -20.3 & -21.3 & -24.0 & -23.7 & -30.3 & -30.2 & -28.5 & -18.8 & -28.6 & -31.2 & -35.3 & -33.8 & -33.9 \\
\hline 25 & & 3.9 & 6.3 & 7.6 & 7.0 & 3.3 & 5 & 3.3 & 9.2 & 11.8 & 16.4 & 19.2 & 32.2 & 45.8 & 58.6 & 71.9 & 82.9 & 88.9 & 94.6 & 90.0 & 89.9 \\
\hline 26 & & 10.0 & 2.6 & 1.4 & 3.3 & 3. & 1. & -0.4 & -1.9 & -0.5 & -2.0 & -1.6 & 5. & 11.4 & 18.1 & 27.8 & 36.3 & 41.1 & 45.5 & 39.7 & 44.6 \\
\hline 27 & & 10.0 & 10.0 & 14.9 & 15.6 & 14.4 & 13. & 11.9 & 6.7 & 6. & 0. & -2 & -3 & -6 & -7.6 & -11.4 & -15 & -14.1 & -17.0 & & -21.6 \\
\hline 28 & & 1.4 & 0.2 & 3.9 & 4. & -0.3 & -5 . & -7.7 & -14.1 & -15.2 & -26.5 & -33.5 & -40.1 & -49.8 & -53.0 & -58.0 & -67.8 & -71.7 & -75.8 & -80.4 & -81.7 \\
\hline 29 & & 7.6 & 5.1 & 7.6 & 8.2 & 5.8 & 6.4 & 5.8 & 3.0 & 4.4 & -2.0 & -4.1 & -2.1 & -3.3 & -1.5 & -1.6 & -4.1 & -4.3 & -7.1 & -5.6 & -8.1 \\
\hline 30 & & 5.1 & 5.1 & 10.0 & 11.9 & 11.9 & 11. & 9.4 & 7.9 & 10.6 & 7.8 & 8. & 15.1 & 20.0 & 21.8 & 30.2 & 33.9 & 33.7 & 33.3 & 36.0 & 31.1 \\
\hline 31 & & 7.6 & 7.5 & 8.8 & 9.4 & 8.2 & 8. & 9.4 & 9.2 & 10.6 & 5.4 & 4.5 & 5.3 & 7.8 & 14.4 & 25.3 & 25.3 & 26.4 & 30.8 & 27.5 & 26.2 \\
\hline 32 & & 0.2 & 2.6 & 6.3 & 13.1 & 14. & 16. & 19.2 & 20.2 & 22 & 22. & 22. & 32. & 35.9 & 43 & & & & & & 71.5 \\
\hline 33 & & 12.5 & 13.7 & 21.0 & 21.7 & 18. & 16. & 15.6 & 15. & 16 & 11.5 & 9. & 6. & 4.1 & 8.3 & 11.8 & 10.6 & 11.7 & 13.7 & 15.2 & 13.9 \\
\hline 34 & & 5.1 & 10.0 & 14.9 & 19.2 & 21.7 & 24.8 & 25.4 & 23.9 & 26.5 & 25.0 & 24.1 & 26.1 & 31.0 & 37.7 & 49.8 & 51.0 & 46.0 & 55.4 & 49.5 & 54.4 \\
\hline
\end{tabular}




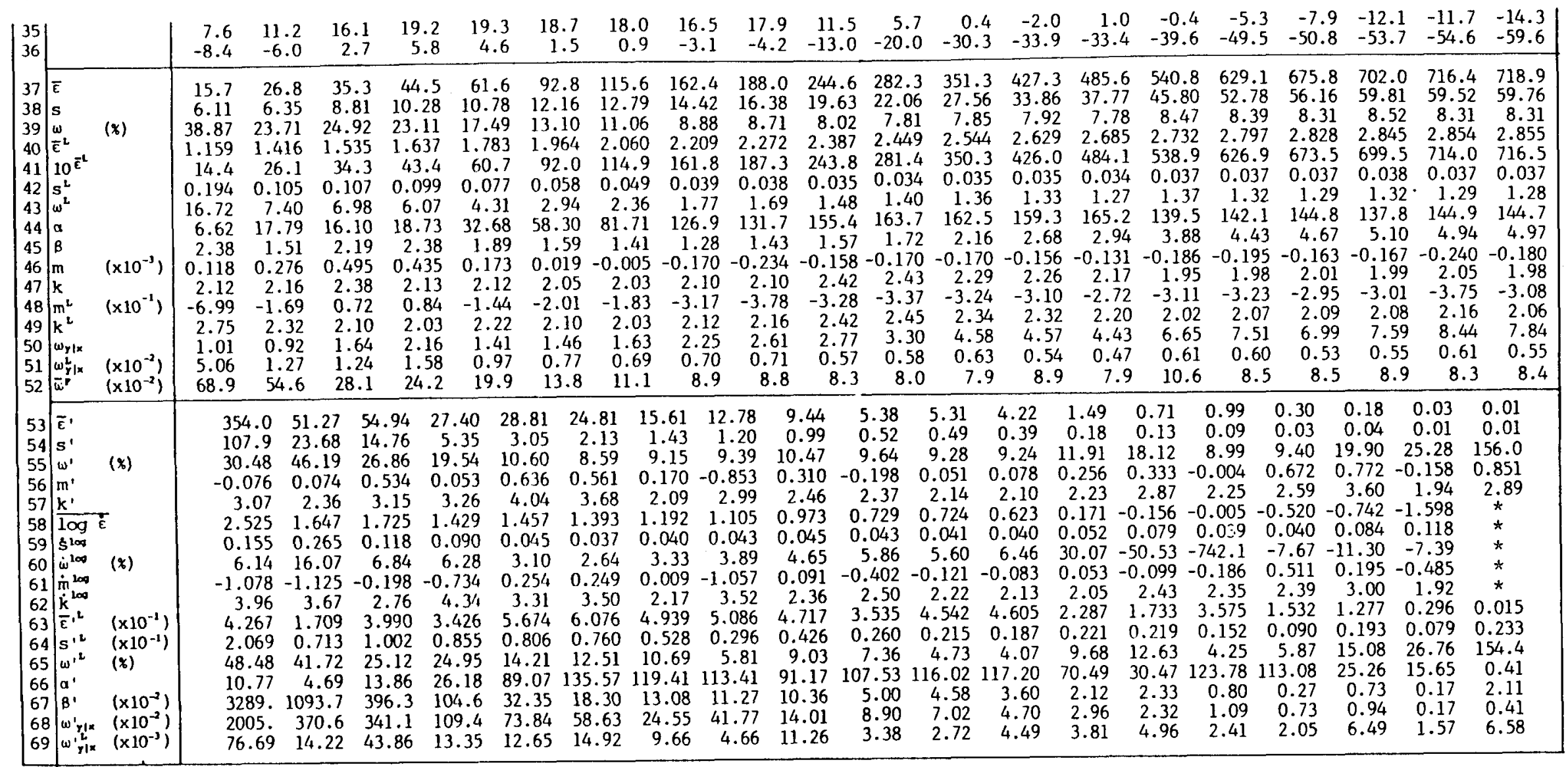

* Some strain rates for this time were negative

$737,624,804,654,720,790,627,758,788,599,755,686,807,761,698,639,710,751,745,793,733,774,704,660, w 1$ th $10^{6} \bar{\epsilon}_{\mathrm{sh}}^{-}=723$. 
TABLE 2-Shrinkage strains measured for individual specimens and their statistics $(\mathrm{D}=160 \mathrm{~mm})\left(\epsilon_{\mathrm{sh}} \times 10^{\circ}\right)$

\begin{tabular}{|c|c|c|c|c|c|c|c|c|c|c|c|c|c|c|c|c|c|c|c|c|}
\hline $\begin{array}{c}\text { time } t \\
\text { (in days) }\end{array}$ & .0104 & 0.042 & 0.142 & 0.313 & 1 & 2.02 & 3 & 6 & 8 & 14 & 21 & 34 & 52 & 91 & 169 & 258 & 412 & 554 & 1105 & 1399 \\
\hline 1 & 1.9 & 1.6 & -0.3 & -1.9 & 2.1 & 3.0 & 3.6 & 4.2 & 3.3 & 5.5 & 3.5 & 5.2 & 7.0 & 3.2 & 2.0 & 5.5 & 4.2 & 4.6 & 3.7 & 2.2 \\
\hline$\frac{1}{2}$ & -1 & -4. & -5.2 & -5.6 & 0.9 & -0.7 & -1 & $\begin{array}{r}4.02 \\
-0.7\end{array}$ & -4.1 & -5. & -9.9 & -11.9 & -15.0 & -22.5 & -32.4 & -42.3 & -43.6 & -50.6 & -53.9 & -56.6 \\
\hline 3 & -3.0 & -4.6 & -6.4 & -10.5 & -17.5 & -17.9 & -17.2 & -17.8 & -18.8 & -17.8 & -18.5 & -16.8 & -13.8 & -15.2 & -17.6 & -16.6 & -16.7 & -12.6 & -9.8 & -11.3 \\
\hline 4 & -3.0 & 1.6 & 0.9 & 4.2 & 7.0 & 5.4 & 2.4 & -1.9 & -5 & 10.5 & 14.8 & 20.5 & -27.3 & -37.3 & -49.5 & -61.9 & -69.4 & -81.2 & -83.3 & -89.7 \\
\hline 5 & 3.2 & 1.6 & 0.9 & 3.0 & 3.4 & 5.4 & 4.9 & 5. & 4. & 6.7 & 7.2 & 8.9 & 10 & 5. & 6.9 & 7.9 & 6.6 & 10.7 & 14.7 & 14.5 \\
\hline 6 & -1.8 & 2.8 & 5.8 & 9. & 19.3 & 23.8 & 24.5 & 27. & 27. & 31. & 35.4 & 35.9 & 42 & 44 & 47.3 & 57.0 & 54.4 & 62.1 & 57.6 & 58.6 \\
\hline 7 & -4.2 & -7.0 & -8.9 & -10.5 & -13.8 & -10.5 & -8. & -5.6 & -5.3 & -1.9 & -0.1 & 0.3 & 4. & -4.2 & 2.0 & 1.8 & -9.3 & 3.3 & 3.7 & 1.0 \\
\hline 8 & -0.5 & -3.3 & -2.8 & -4.3 & -5.2 & -5.6 & -7.4 & -9.2 & -10.2 & -10.5 & -13.6 & -15.6 & -16.2 & -20.1 & -22.5 & -23.9 & -24.0 & -28.5 & -28.2 & -32.1 \\
\hline 9 & -4.2 & 0.4 & 3.4 & 3.0 & 5.8 & 6. & 7.3 & 9.1 & 10.6 & 15.3 & 17.0 & 18.7 & 26 . & 32 & 32.6 & 38 & 45.8 & 46.2 & 45.3 & 45.1 \\
\hline 10 & 6.8 & 15.1 & 20.5 & 22.6 & 20.5 & 28.7 & 31.8 & 37.3 & 38. & 44. & 46 & 49.3 & 56 & 58. & 62. & 63.1 & 60.5 & 65 & 63.7 & 64.7 \\
\hline 11 & -5.4 & -9.5 & -11.3 & -17.8 & -23.6 & -22.8 & -20.9 & -22.7 & -23.7 & -25.2 & -27 & -31.5 & -56 & -48 & -39 & -43.5 & -42.4 & -48.1 & -45.3 & -50.5 \\
\hline 12 & -7 & 5.3 & 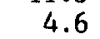 & -1.9 & -7.7 & -3.2 & -6.2 & -4.3 & -1 & 3.0 & 7.2 & 13.8 & 19 & 27.7 & 31.4 & 41.0 & 47.1 & 52.3 & 52.7 & 45.1 \\
\hline 13 & .8 & 10.2 & 11.9 & 12.8 & 13.2 & 16.5 & 11.0 & 11. & 13. & 12 & 12.1 & 16 & 16 & 24. & 17 & 19.0 & 31.1 & 8 & 18.4 & 18.1 \\
\hline 14 & -5.4 & -7.0 & -6 . & -4. & -2.8 & -1 & -3.7 & -6 & -6 & -6 & -7.5 & -5 & -5 & -6 & -7.8 & -11.7 & -14.2 & -17 & -20.8 & -23.5 \\
\hline 15 & -1.8 & -2.1 & -4.0 & -6.8 & -4.0 & -6.8 & -7.4 & -6. & -6 . & -4.3 & -3.8 & -2.1 & 2. & 4.4 & 11.8 & 16.5 & 21.3 & 24.2 & 22.1 & 24.3 \\
\hline 16 & 3.2 & -3.3 & -6.4 & -9.2 & -11.3 & -11.7 & -14.7 & -17 & -18 & -17.8 & -17.3 & -18.1 & -21.1 & -22.5 & -26.2 & -30.0 & -35.0 & -34.7 & -38.0 & -38.2 \\
\hline $17 \varepsilon_{n}-\bar{\varepsilon}_{\text {m }}$ & -0.5 & -7.0 & -7.7 & -6.8 & -4.0 & -5.6 & -8.6 & -12.9 & -15. & -17.8 & -18.5 & -20.5 & -21.1 & -21.3 & -25.0 & -28.8 & -32.6 & -32.2 & -33.1 & -34.6 \\
\hline 18 an & 1.9 & 4.0 & 0.9 & -4.3 & -8.9 & 6.8 & -7.4 & -3.1 & -2 . & -0.7 & 2.3 & & 10.7 & 21.6 & 24.0 & 30.0 & 40.9 & 43.8 & 45.3 & 48.8 \\
\hline 19 & -4.2 & -10.7 & -10.1 & -12.9 & -17.5 & -16.6 & -19.6 & -23.9 & -24 & -30 & -33.2 & -37.7 & -42.0 & -45.8 & -53.2 & -59.5 & -63.2 & -70 & -73.5 & -75.0 \\
\hline 20 & 1.9 & 16.3 & 19.3 & 17.7 & 15.6 & 18.9 & 17.1 & 16.5 & 15. & 15.3 & 14.6 & 12.6 & 11.9 & 9.3 & 6.9 & 3.0 & 1.7 & -5.3 & -7.4 & -8.8 \\
\hline 21 & -4.2 & -9.5 & -7.7 & -8.0 & -12.6 & -13.0 & -14.7 & -15.4 & -15 . & -11 & -8.7 & -5.8 & -1 & 4. & 9.3 & 15.3 & 17.6 & 20.5 & 19.6 & 23.0 \\
\hline 22 & 4.4 & 10.2 & 10.7 & 14.0 & 11.9 & 21.4 & 24.5 & 31.2 & 32 & 38 & 40.3 & 42 & 48.7 & 51. & 53.4 & 54 & 53.2 & 53 & 55.1 & 56.1 \\
\hline 23 & 07 & -0.9 & -1 & -0.7 & -2 & -0 . & -3.7 & -5. & -6 . & -8 & -6 & -8.3 & -6 & -5 . & -1.7 & 0.6 & 7.8 & 0 & 9.8 & 10.8 \\
\hline 24 & 3.2 & 0.4 & -0.3 & 3.0 & 5.8 & 4.2 & 6.1 & 6. & 8. & 6 & 7.2 & 6 & 0 & -0 . & -5.4 & -10.4 & -13.0 & -15.1 & -6.1 & -6.4 \\
\hline 25 & -1.8 & 2.8 & 4.6 & 12.8 & 18.1 & 16.5 & 18.3 & 20. & 19. & 21 & 23.1 & 22 & 24.2 & 26.5 & 28.9 & 30.0 & 29.9 & 30.3 & 39.2 & 41.4 \\
\hline 26 & -0.5 & 1.6 & 0.9 & 1.8 & 3.4 & 0.5 & 2.4 & 4. & 11. & 4.2 & 7.2 & 10.1 & 10.7 & 13.0 & 15.4 & 20.2 & 23.8 & & 28 & 31.6 \\
\hline 27 & -3.0 & -7.0 & -6.4 & -6.8 & -6.4 & -13.0 & -14.7 & -17. & -17 & -21 & -22.2 & -25.4 & -27.3 & -32.4 & -33 & -32 & -32.6 & -35 & -39.2 & -37.0 \\
\hline 28 & 3. & 7.7 & 13.2 & 14.0 & 11.9 & 10.3 & 12.2 & 10. & 9. & 4.2 & -0.1 & -2.1 & -5.2 & -9.1 & -12.7 & -14.1 & -17.9 & -16.3 & -14.7 & -11.3 \\
\hline 29 & -3.0 & -7.0 & -8.9 & -9.2 & -8.9 & -3.2 & 3.6 & 11.6 & 14. & 20.2 & 25 & 32.2 & 35.2 & 43.6 & & & & & 74.8 & 75.7 \\
\hline 30 & 1.9 & 0.4 & -2.8 & -4.3 & -2.8 & -8 & -7.4 & -10 & -12 & -14.1 & -14 & -15.6 & -18.7 & -21.3 & -20.1 & -20.2 & -19.1 & -13.8 & -17.2 & -16.2 \\
\hline 31 & 1.9 & -0.9 & -4.0 & -3.1 & -4.0 & -9. & -8.6 & -9.2 & -9. & -10.5 & -12.4 & -11.9 & -12.6 & -14.0 & -14.0 & -16.6 & -20.3 & -20.0 & -17.2 & -13.7 \\
\hline 32 & 4.4 & 6.5 & 8.3 & 7.9 & 8.3 & 4. & 7.3 & 9.1 & & $\mathbf{b}$ & & 4 & 0 & -0 & & & 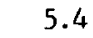 & -0 & -4.9 & -2.7 \\
\hline 33 & 5.6 & 5.3 & 7.0 & 10.4 & 13.2 & 7.9 & 8.5 & 4.2 & 3.3 & -0.7 & -2.6 & -5.8 & -10.1 & -11.5 & -11.5 & -14.1 & -16.7 & -16.3 & -12.3 & -10 \\
\hline
\end{tabular}




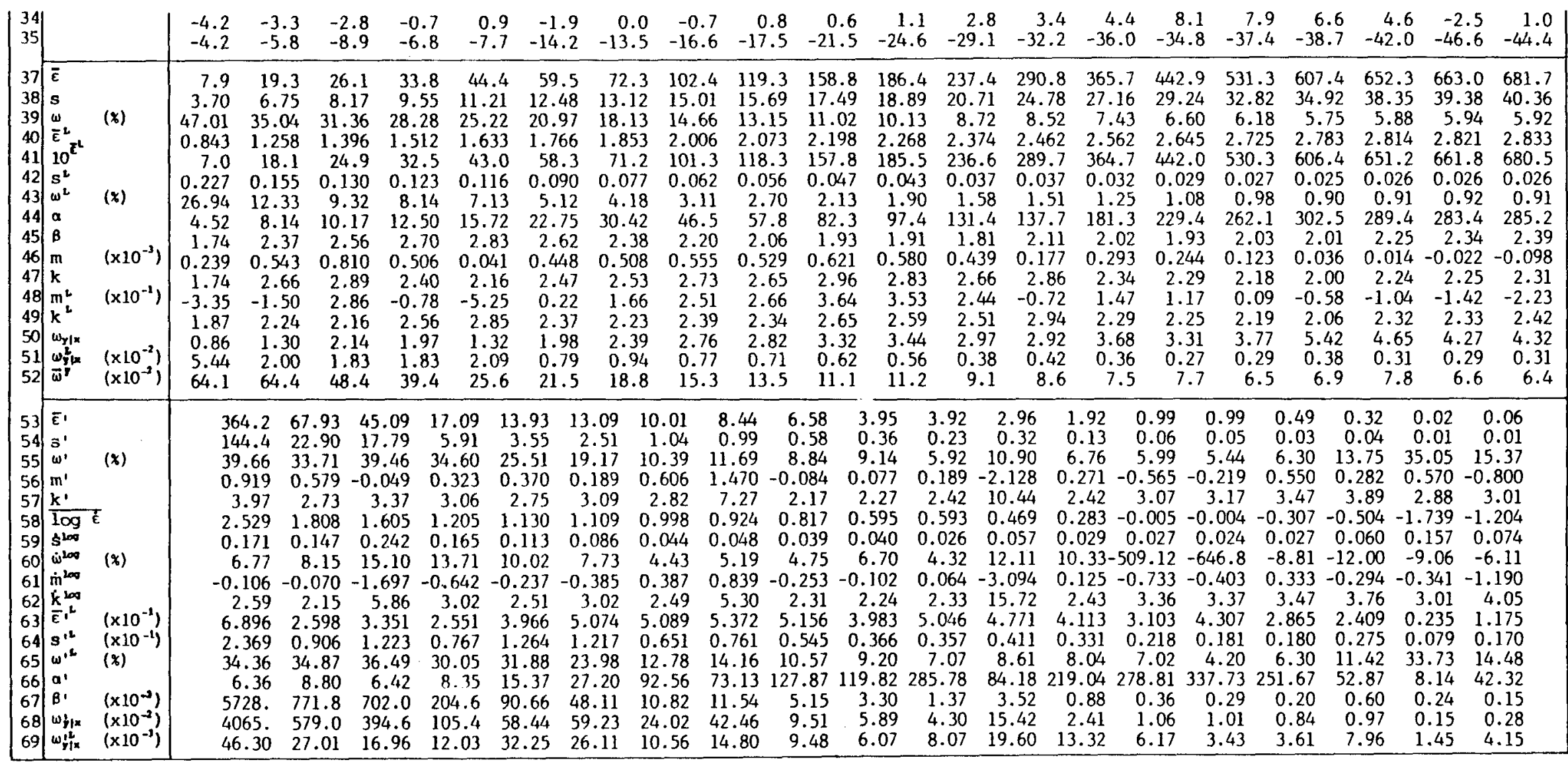

Nore: Just before printing, at $t=1553$ days, $10^{6} \mathrm{col}=688,631,679,595,706,748,674,657,730,750,637,729,723,666,712,658,652,744$,

$617,674,708,745,699,686,728,717,649,679,761,673,671,681,677,686,646$, w1th $10^{6} \bar{\epsilon}_{\mathrm{sh}}=688$. 


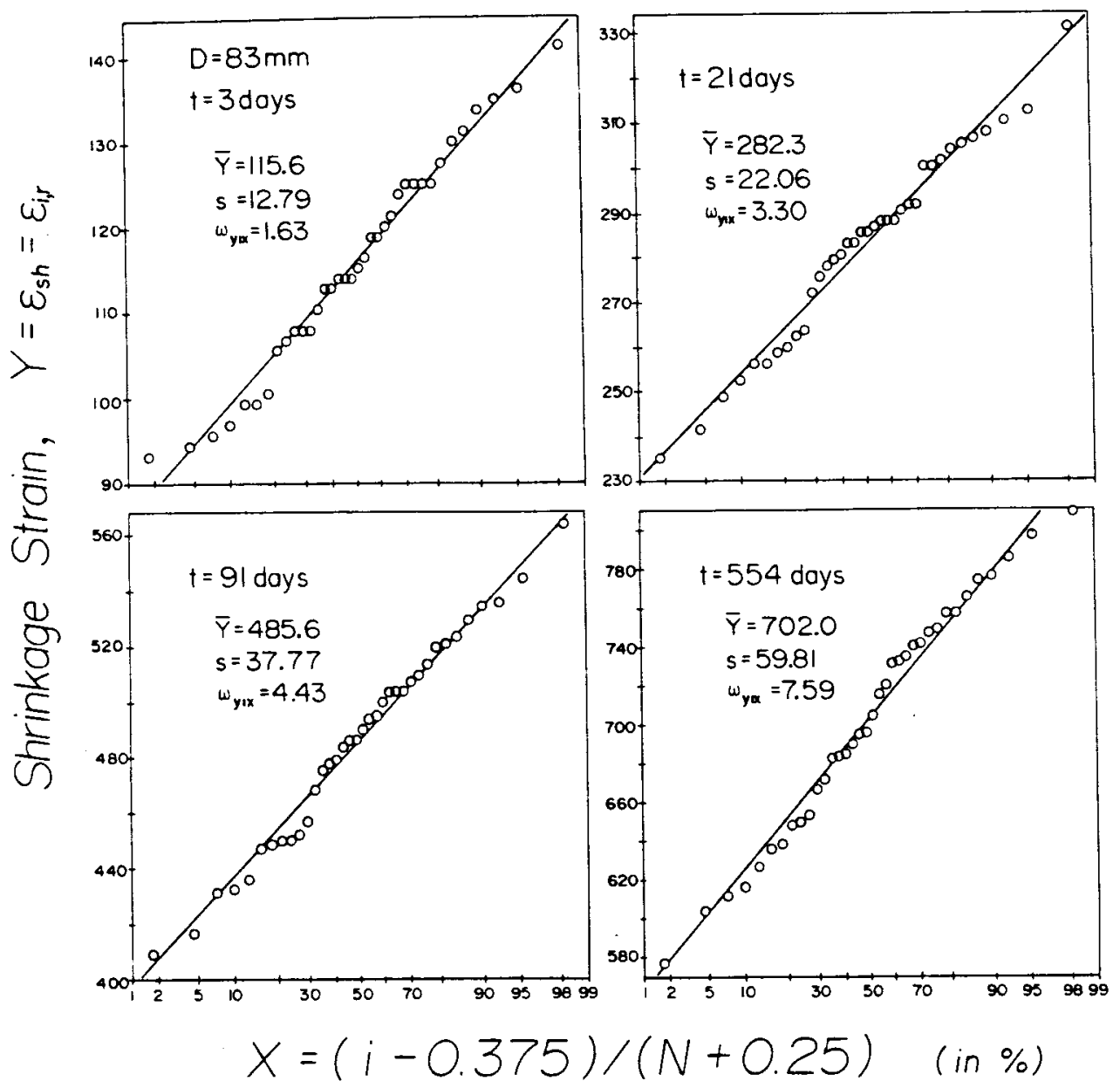

FIG. 2-Cumulative distributions of shrinkage strain for small diameter specimens.

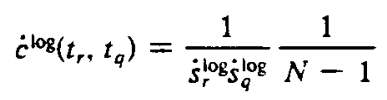

$$
\begin{aligned}
& \sum_{i=1}^{N}\left(\log \dot{\epsilon}_{i, r}-\overline{\log \dot{\epsilon}_{r}}\right)\left(\log \dot{\epsilon}_{i, q}-\overline{\log \dot{\epsilon}_{q}}\right)
\end{aligned}
$$

Their values are plotted in Fig. 15.

Another interesting statistic is the ratio of the random scatter of a single specimen to the random scatter of all the specimens combined. To define the random scatter of a single specimen we must know the smoothing formula for shrinkage, which we consider as $[4,12]$

$$
\epsilon_{s h}=\epsilon_{\infty}\left[1+\left(\frac{\tau_{s h}}{t}\right)^{r}\right]^{-1 / 2 r}
$$

where

$$
\begin{aligned}
\epsilon_{\infty}, \tau_{s h}, \text { and } r & =\text { empirical constants, } \\
t & =\text { duration of drying, } \\
\epsilon_{\infty} & =\text { final shrinkage, and } \\
\tau_{s h} & =\text { shrinkage half-time, usually } r=0.4 \text { to } 1.0
\end{aligned}
$$

This formula, in contrast to some others, satisfies two requirements of diffusion theory [4,13]: (1) for short $t$ it reduces to $\epsilon_{s h}-$ $\sqrt{t}$; and (2) the size dependence can be introduced as the dependence of $\tau_{\text {sh }}$ on size-square. Since the shrinkage law is not know'n exactly, the use of Eq 9 inevitably entails some error. This error should be small if the formula is theoretically well justified. Anyhow, there is no way to eliminate the error of the smoothing formula per se.

The series of shrinkage strains $\epsilon_{i, r}$ for each specimen number $i$ was optimally fitted by $\mathrm{Eq} 9$, minimizing the $\operatorname{sum} \Sigma_{i}\left(\epsilon_{i, r}-\epsilon_{i, r}^{F}\right)^{2}$

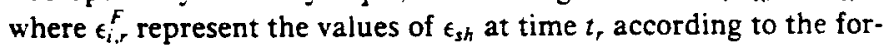
mula ( $\mathrm{Eq} 9$ ) obtained from the parameter values $r, \epsilon_{\infty}$, and $\tau_{s h}$ that were found to be optimum for specimen number $i$. The random scatter for specimen number $i$ over its entire history $(r=1, \ldots$, $n$ ) is then characterized by the standard deviation

$$
s_{i}=\left[\frac{1}{n-2} \sum_{r=1}^{n}\left(\epsilon_{i, r}-\epsilon_{i, r}^{F}\right)^{2}\right]^{1 / 2}
$$

We use here $n-2$ instead of $n$ because the problem is essentially equivalent to linear regression (in which two data values can be 


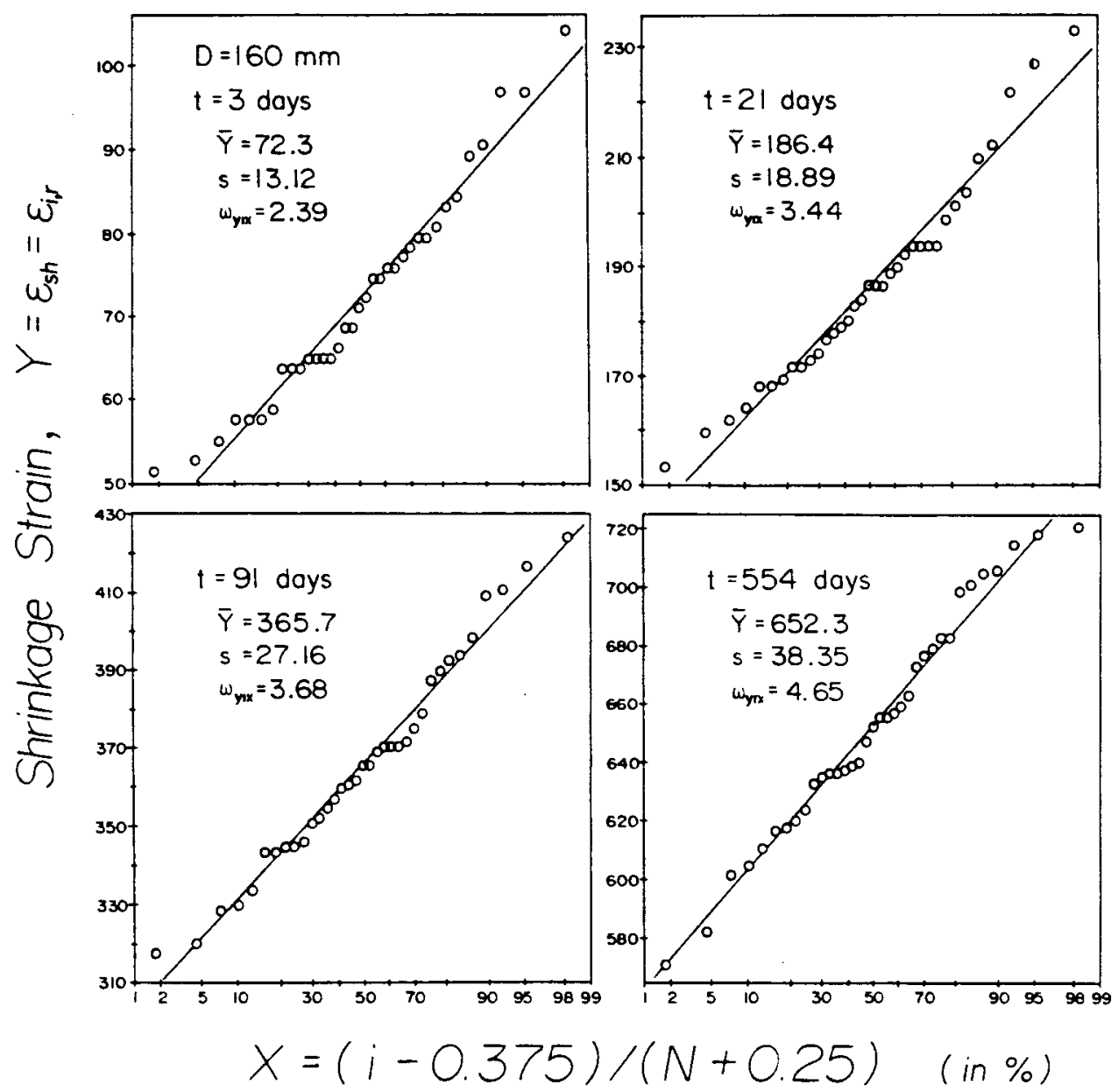

FIG. 3-Cumulative distributions of shrinkage strain for large diameter specimens.

fitted perfectly, and so only the further ones determine scatter). The average standard deviation of a single specimen may then be defined as

$$
s^{*}=\left(\frac{1}{N} \sum_{i=1}^{N} s_{i}^{2}\right)^{1 / 2}
$$

For comparison we also calculate the standard deviation character. izing the overall scatter of the data set for all times $t$, and all specimens $i=1, \ldots, N$

$$
s=\left[\frac{1}{n N-2} \sum_{r=1}^{n} \sum_{i=1}^{N}\left(\epsilon_{i, r}-\epsilon_{r}^{F}\right)^{2}\right]^{1 / 2}
$$

in which $\epsilon_{r}^{F}$ is the value of the smoothing formula (Eq 6) obtained by fitting all the specimens combined. The values of $s$ and $s^{*}$ for the entire period of measurements are listed in Table 4 under the column labeled as $0 \leq \hat{t} \leq 1399$ days. Since the magnitude of scatter evolves with time, we also show in Table 4 the values of $s$ and $s *$ calculated from the data for limited time periods as indicated.
The random scatter of a single specimen and of the entire group of specimens can be also characterized on the basis of $\log \epsilon_{i, r}$ instead of $\epsilon_{i, r}$. We then have the standard deviations

$$
\begin{gathered}
s_{i}^{L}=\left[\frac{1}{n-2} \sum_{r=1}^{n}\left(\log \epsilon_{i, r}-\log \epsilon_{i, r}^{F}\right)^{2}\right]^{1 / 2} \\
s^{* L}=\left(\frac{1}{N} \sum_{i=1}^{N} s_{i}^{L^{2}}\right)^{1 / 2} \\
s^{L}=\left[\frac{1}{n N-2} \sum_{r=1}^{n} \sum_{i=1}^{N}\left(\log \epsilon_{i, r}-\log \epsilon_{r}^{F}\right)^{2}\right]^{1 / 2}
\end{gathered}
$$

The values of $s_{i}^{L}$ and $s^{* L}$ for the entire group and for a single specimen are again shown in Table 4 for the entire time range of measurements, as well as for limited periods. Table 4 further lists the ratios $s^{*} / s$ and $s^{* L} / s^{L}$ which characterize the ratio of a single specimen scatter to the group scatter.

The deviations of the smoothed single-specimen shrinkage from the smoothed shrinkage values for the entire group are $\epsilon_{i, r}^{F}-\epsilon_{r}^{F}$, 


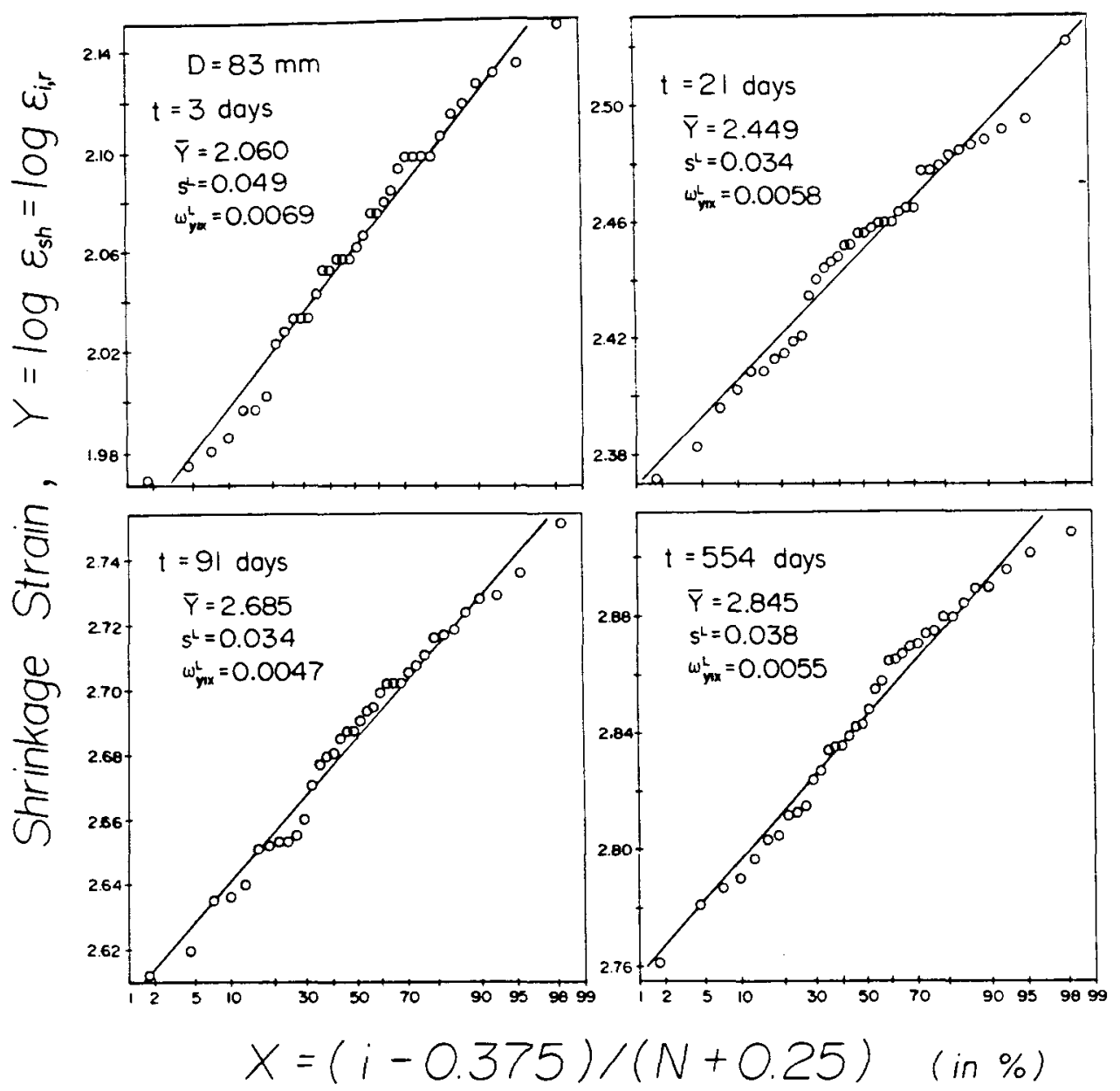

FIG. 4-Cumulative distributions of log-strain for small diometer specimens.

and they may be characterized by the standard deviations and coefficients of variation

$$
\bar{s}_{r}=\left[\frac{1}{N-2} \sum_{i=1}^{N}\left(\epsilon_{i, r}^{F}-\bar{\epsilon}_{r}^{F}\right)^{2}\right]^{1 / 2}, \quad \bar{\omega}_{r}=\frac{\bar{s}_{r}}{\epsilon_{r}^{F}}
$$

which may be calculated for each time $t_{r}$. For all times $t_{r}(r=1$, $2, \ldots, n$ ) combined

$$
\bar{s}=\left(\frac{1}{n} \sum_{r=1}^{n} \tilde{s}_{r}^{2}\right)^{1 / 2}, \quad \bar{\omega}=\left(\frac{1}{n} \sum_{r=1}^{n} \bar{\omega}_{r}^{2}\right)^{1 / 2}
$$

The values of $\tilde{s}_{r}, \tilde{s}$, and $\bar{\omega}$ are also shown in Table 4 for the entire measurement period as well as limited periods. The ratio $\bar{s} / s$ (Table 4 ) indicates the portion of statistical variability due to the randomness of permanent specimen properties acquired at its casting. So the value $1-(\tilde{s} / s)$ could then be regarded as the portion of scatter that is caused by the randomness of the shrinkage process in time.
The scatter of the data relative to their mean was already characterized by the values of $\omega$ on line 39 of Tables 1 and 2 . The scatter of the data relative to the smoothing formt la ( $\mathrm{Eq} 9$ ) may be characterized by the coefficient of variation

$$
\bar{\omega}_{r}^{F}=\frac{1}{\bar{\epsilon}_{r}}\left[\frac{1}{N-1} \sum_{i=1}^{N}\left(\epsilon_{i, r}-\epsilon_{r}^{F}\right)^{2}\right]^{1 / 2}
$$

where $\epsilon_{r}^{F}$ is the smoothed value for all the specimens according to the optimum fit by Eq 9 (see Tables 1 and 2, line 52).

The evolution of statistical scatter in time is further illustrated in Figs. 14, 16, 17. Figure 14 shows the history of all specimens in one plot (that is, the plot of the values of $\epsilon_{s h}$ according to lines 1-36 of Tables 1 and 2). Figures 16 and 17 (for two specimen diameters $D$ ) show the evolution of the deviation from the group mean, for both $\epsilon_{s h}$ and $\log \epsilon_{s h}$, and for various types of mean (mean of $\epsilon_{s h}$ or $\log \epsilon_{s h}$ from all specimens used). On the bottom of Figs. 16-17, one can see the evolution of the deviations from the optimum fit by the formula (Eq 9). 


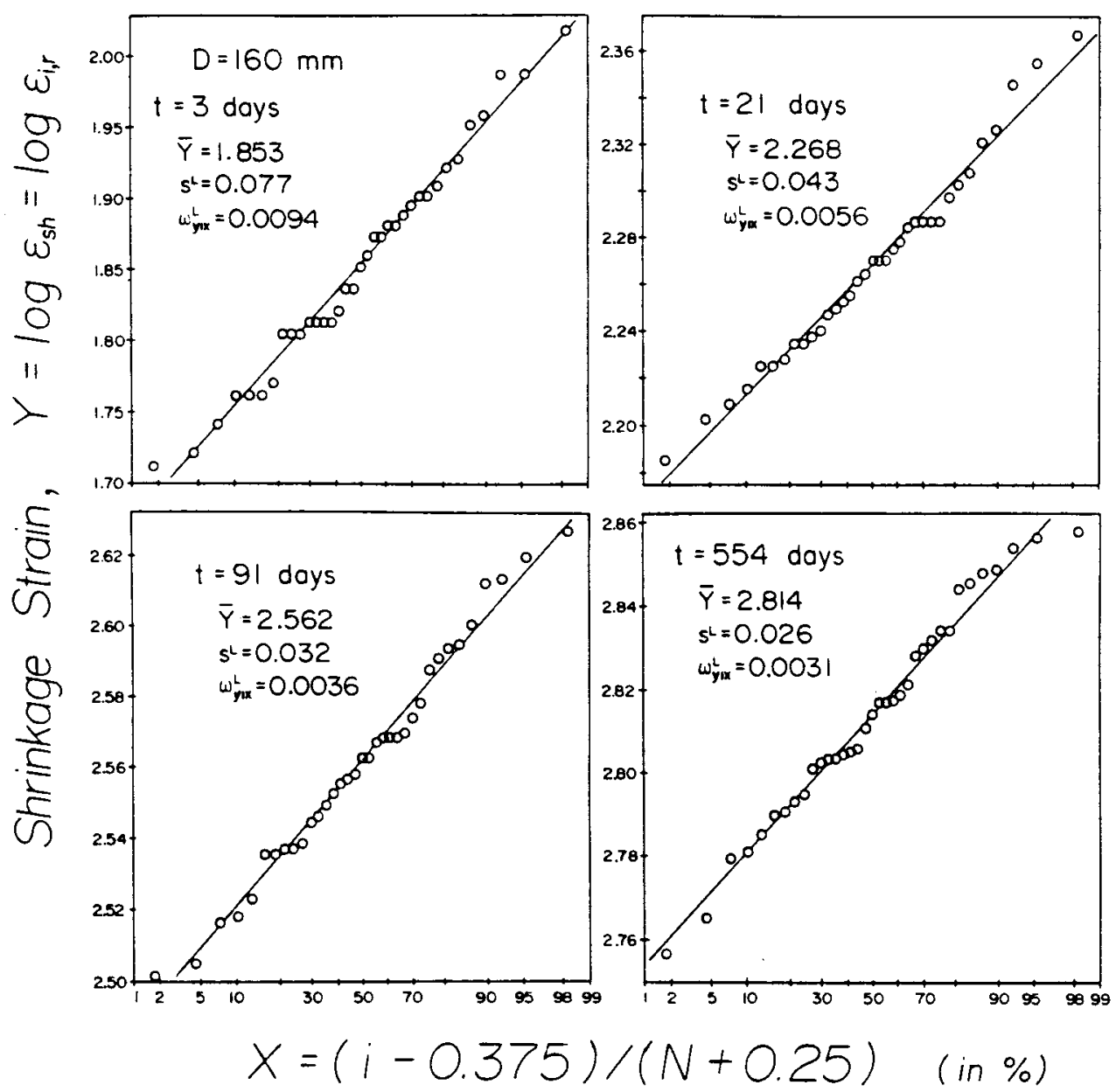

FIG. 5-Cumulative distributions of log.strain for large diameter specimens.

\section{Observations from Test Results}

1. While the standard deviation of shrinkage values of all specimens increases with time, their coefficient of variation decreases and appears to reach a constant value of about $8.5 \%$ for the cylinders of smaller diameter, and about $6 \%$ for the cylinder of larger diameter (Tables 1 and 2, Figs. 16-17). The observed means for the set of all specimens have corresponding coefficients of variation of only 1.4 and $1.0 \%$. The values 8.5 and $6 \%$ are relatively small, which reveals that the shrinkage process per se is not a major source of uncertainty. Rather it is the various random, poorly controlled, or unknown influences which make shrinkage predictions in practice as uncertain as they are known to be $[1,4,5]$.

2. The distribution of shrinkage values at a fixed time may be approximately considered as normal (Gaussian), as confirmed by Figs. 2-5 and Figs. 10-11. However, the log-normal distribution and the gamma distribution fit the observed frequency density distributions (Figs. 10-11) at least just as well, and they are both obtained to be very close to the normal distribution. The reason for this closeness is that the coefficient of variation is relatively small.
3. There are nevertheless small systematic deviations from the normal distribution. All the observed distributions of shrinkage strain are slightly asymmetric and have a positive skewness $m$ (line 46 of Tables 1,2 ). This means that they are all skew to the right, that is, have an extended tail toward the high values and a short tail toward the small values (for normal distribution $m$ would be zero). The positive value of skewness confirms that asymmetric distributions such as log-normal or gamma are better than the normal distribution. This agrees with the fact that negative shrinkage values are impossible. The log-normal and gamma distributions, unlike the normal distribution, have the advantage that they do not permit negative values.

4. Another deviation from normal distribution is due to the fact that all the observed distributions are slightly platykurtic (that is, have a flat top and short tails). This is revealed by the fact that the kurtosis coefficient $k$ is systematically less than 3 (the value of kurtosis for normal distribution) (Tables 1,2 ).

5. The observed cumulative distributions of shrinkage rates (Figs. 6-7) show somewhat larger deviations from the normal distribution, with a more pronounced skewness. Mostly they deviate 


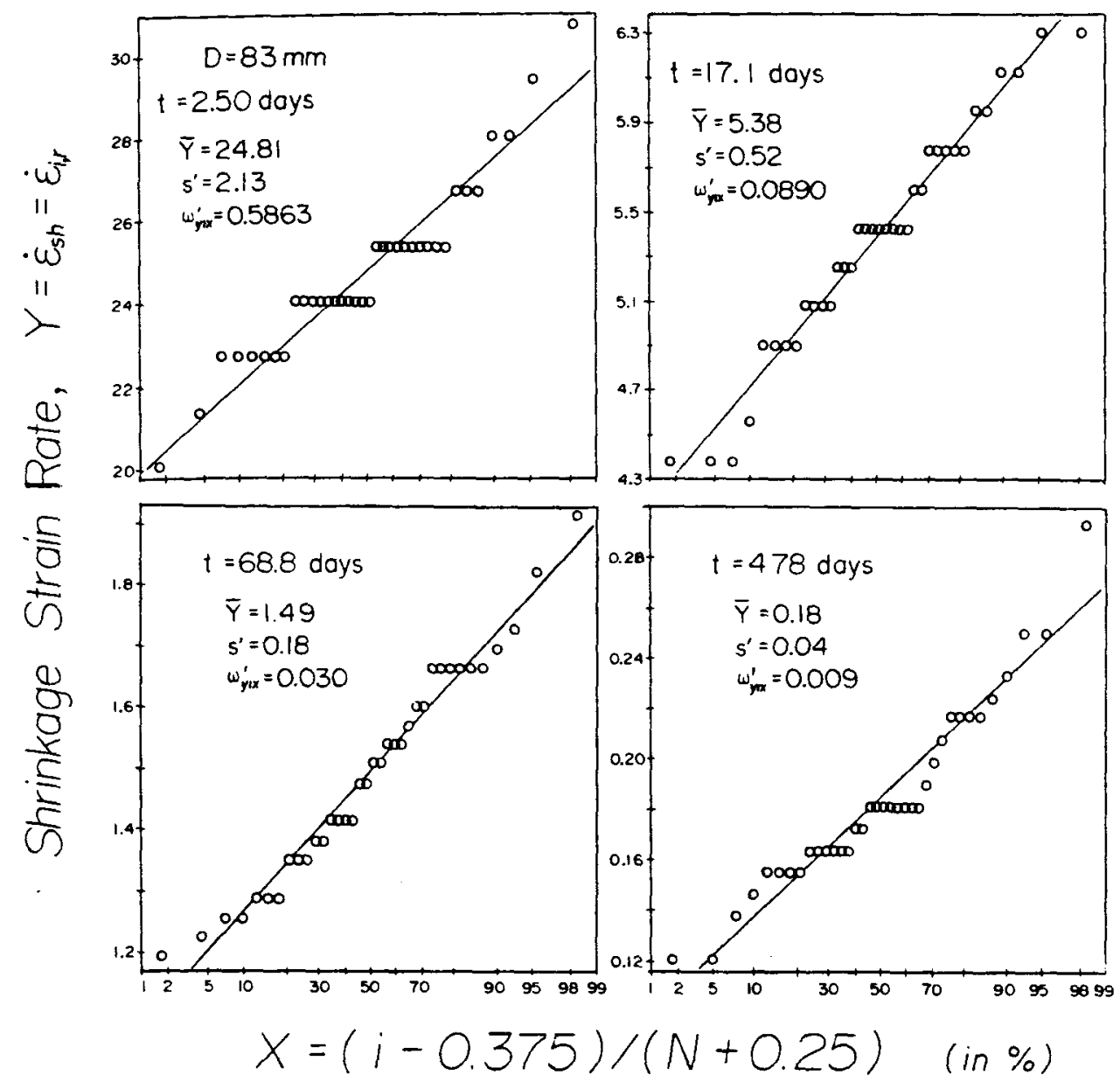

FIG. 6-Cumulative distributions of shrinkage strain rate for small diameter specimens.

upward from the straight line at the right end (Figs. 6-7), which indicates skewness to the right. The skewness coefficients $m^{\prime}$ for the shrinkage rates are larger than those for the shrinkage values (see lines 46 and 56 of Tables 1 and 2). The observed cumulative distributions of $\log \dot{\epsilon}_{i, r}$ and the frequency distributions of the observed rates (Figs. 12-13) also reveal skewness. Thus, the shrinkage rates are described somewhat better by the gamma or log-normal distributions than by the normal distribution, although the normal distribution seems also acceptable empirically (theoretically, though, it is objectionable because it permits negative shrinkage rates which are impossible). Similar conclusions ensue from Figs. 8-9 for log-rates.

6. The deviations of a single-specimen shrinkage from the group mean observed at various times are correlated. For short time intervals the correlation is high, and for long intervals the correlation significantly decreases (Fig. 15), with the correlation coefficient $c$ of these deviations being about 0.6 for the time interval from 3 to 550 days. The correlation of the deviations of the singlespecimen shrinkage rates from the group mean is much weaker (Fig. 15).

7. In agreement with the foregoing observation, a specimen whose shrinkage is on the high (or low) side of the mean is likely to remain on the high (or low) side for short times, but less likely for longer times. This is illustrated by the histories of shrinkage deviations shown in Figs. 16-17.

8. The standard deviation of the individual specimen shrinkage values from the smoothed shrinkage curve of the same specimen is much smaller than (about one third of) the standard deviation of the individual shrinkage values from the mean for the group of all specimens.

9. Observations $5-8$ suggest that the shrinkage process of one specimen would be better modeled as a nonstationary random process with independent increments (a model assumed in Ref 17), rather than as a process with independent random total values.

\section{Acknowledgment}

Financial support was provided by the Swiss Federal Institute of Technology for the conduct of the measurements in Lausanne, and by the U.S. National Science Foundation (under Grant FED 7400 to Northwestern University) for the statistical analysis in Evanston. 


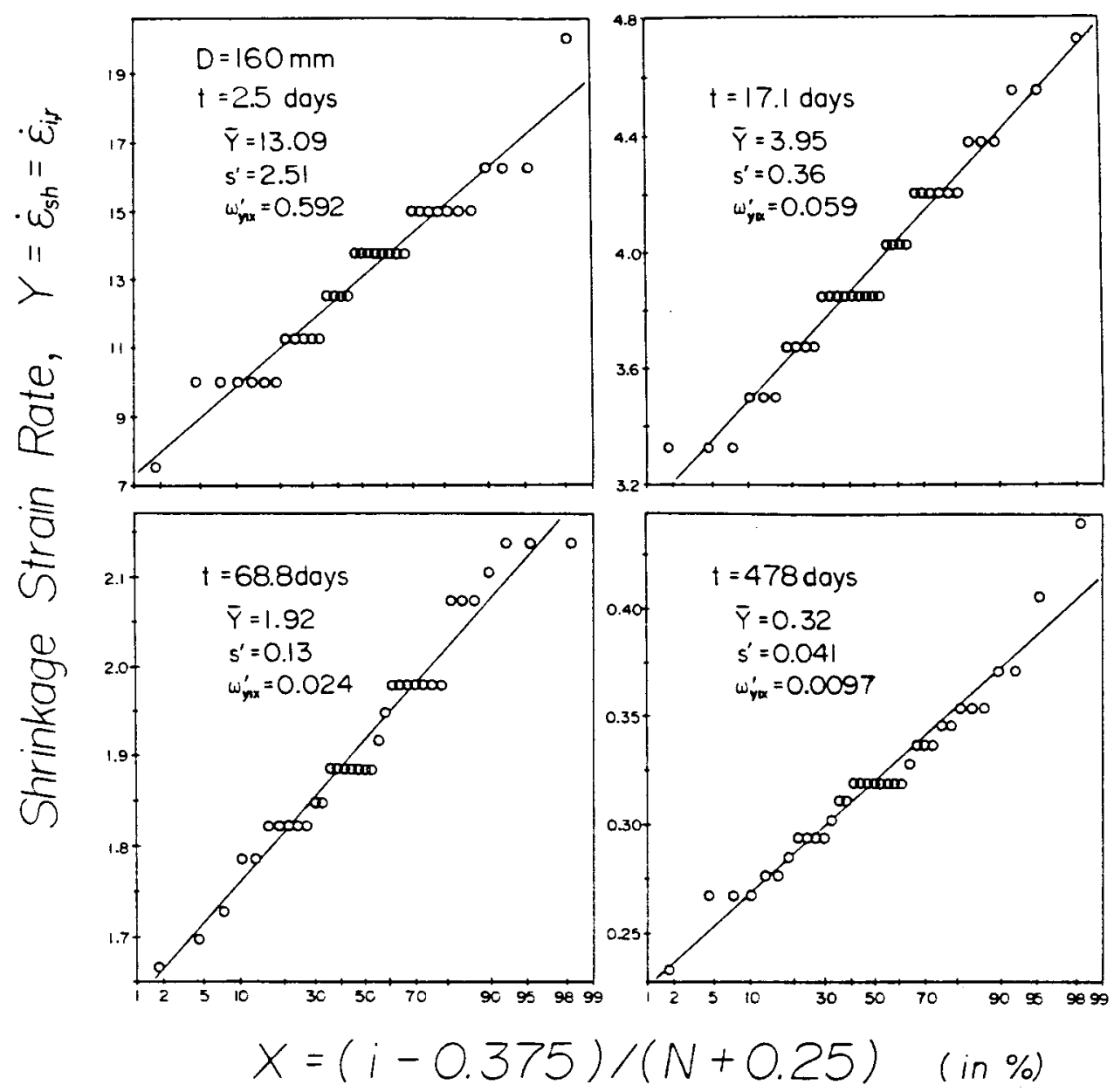

FIG. 7-Cumulative distributions of shrinkage strain rate for large diameter specimens. 

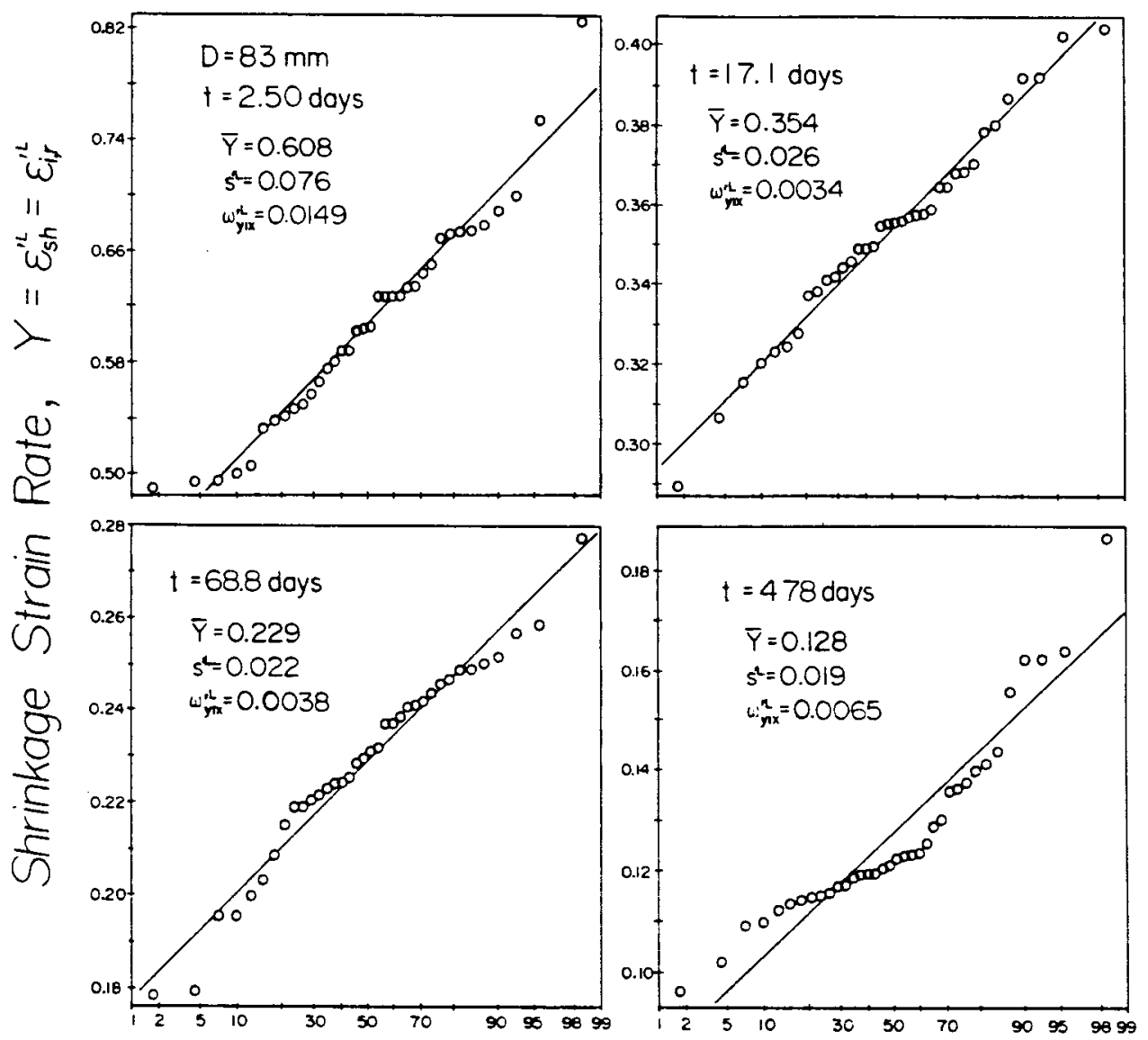

$$
x=(i-0.375) /(N+0.25) \quad(\text { in } \%)
$$

FIG. 8-Cumulative distributions of the rate of log-strain for small diameter specimens. 


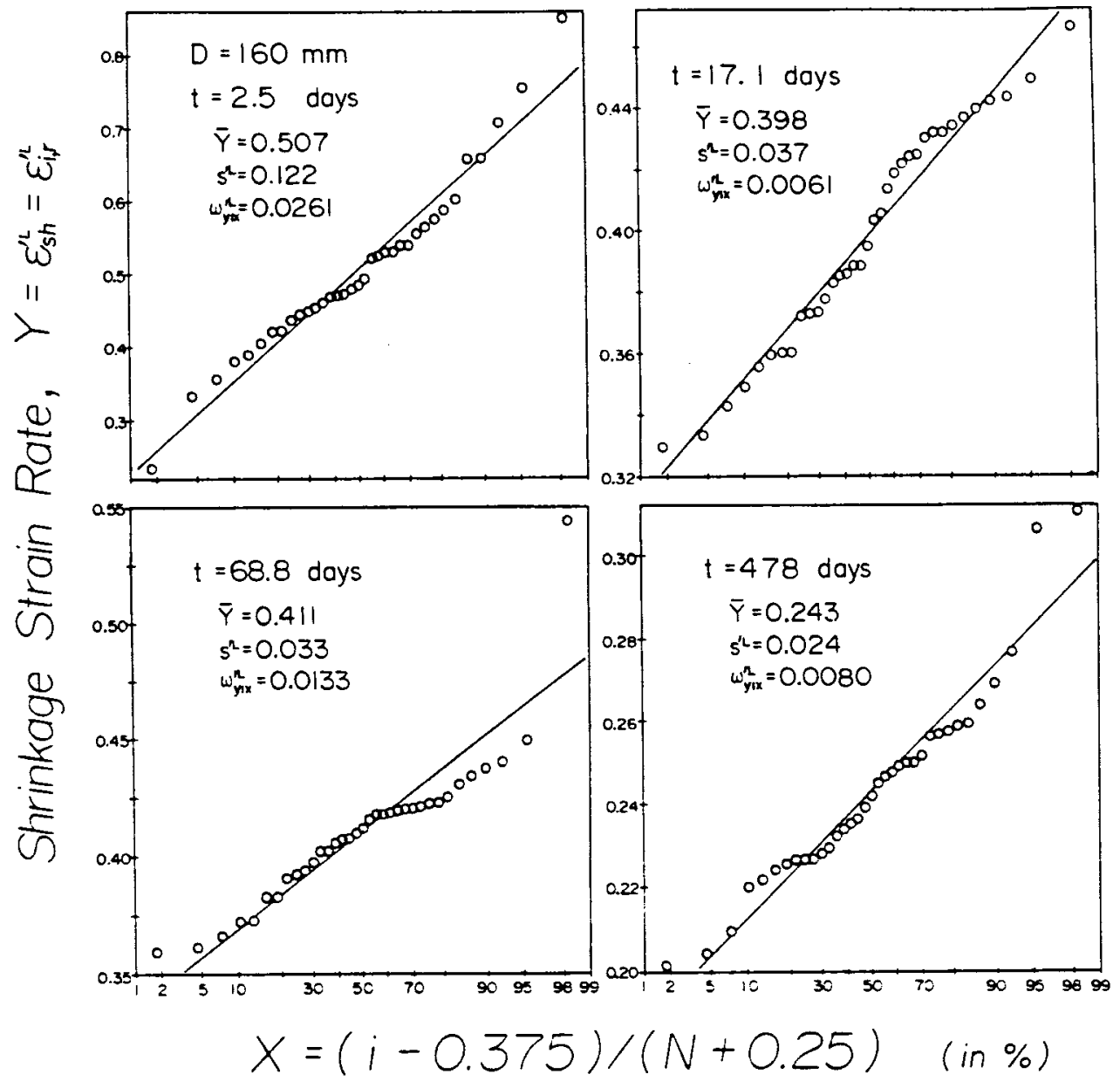

FIG. 9-Cumulative distributions of the rate of log-strain for large diameter specimens. 

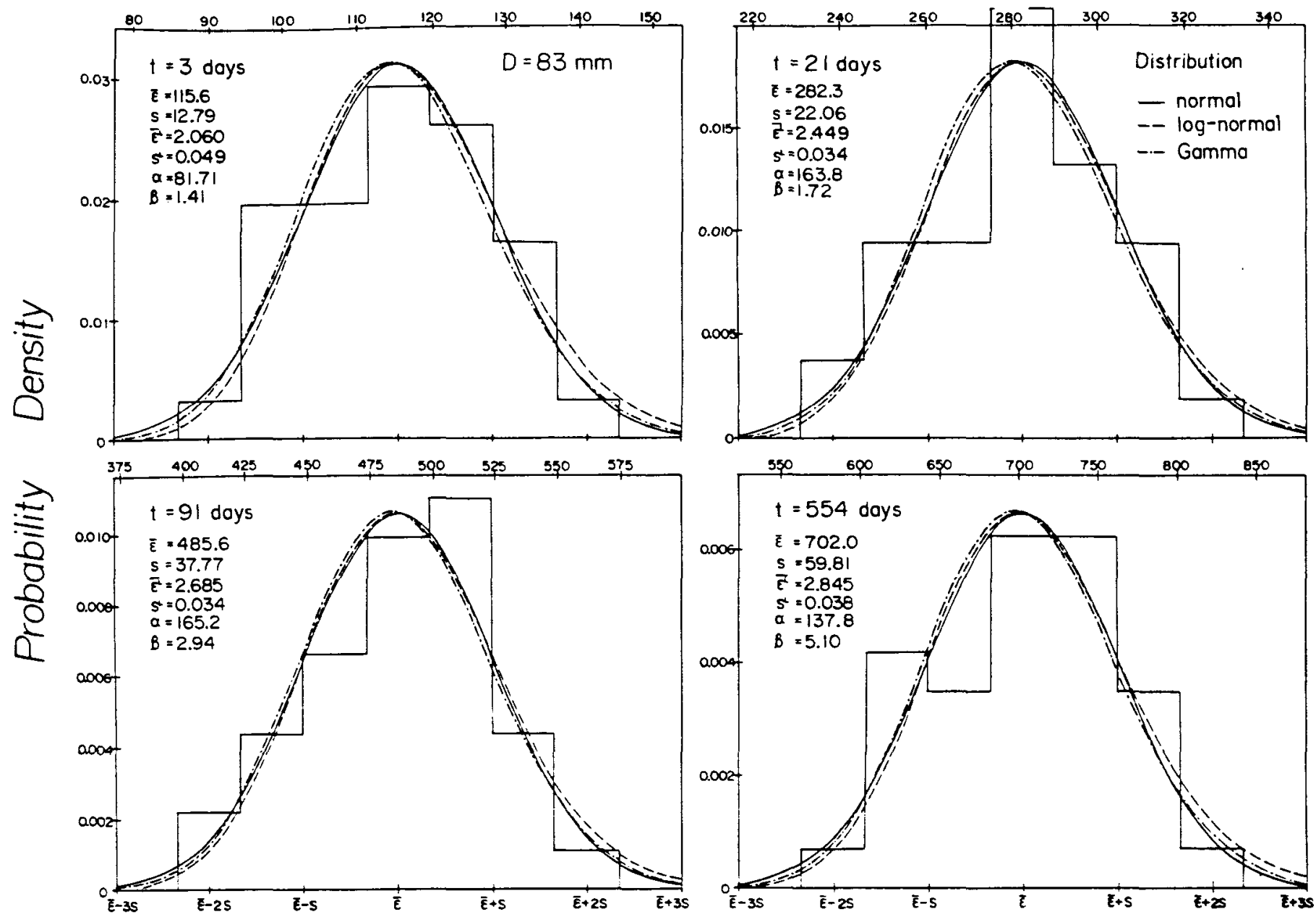

Shrinkage Strain, $\varepsilon_{s h}=\varepsilon_{i, r}\left(\times 10^{\circ}\right)$

FIG. 10-Probability density distributions of shrinkage strain and its optimum fits by normal, log-normal, and gamma distributions for small diameter specimens. 


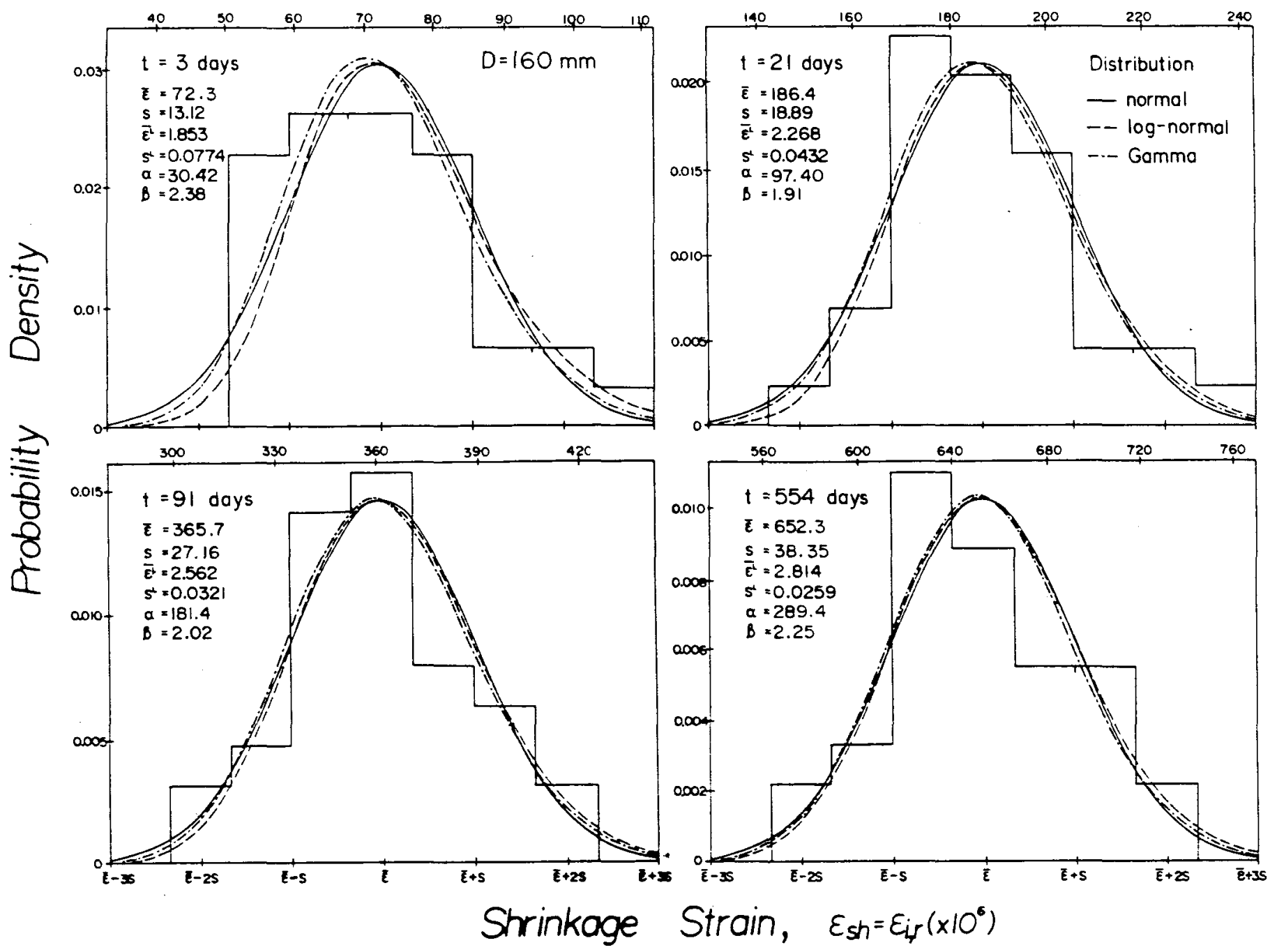

FIG. 11-Probability density distributions of shrinkage strain and its optimum fits by normal. log-normal, and gamma distributions for large diameter specimens. 

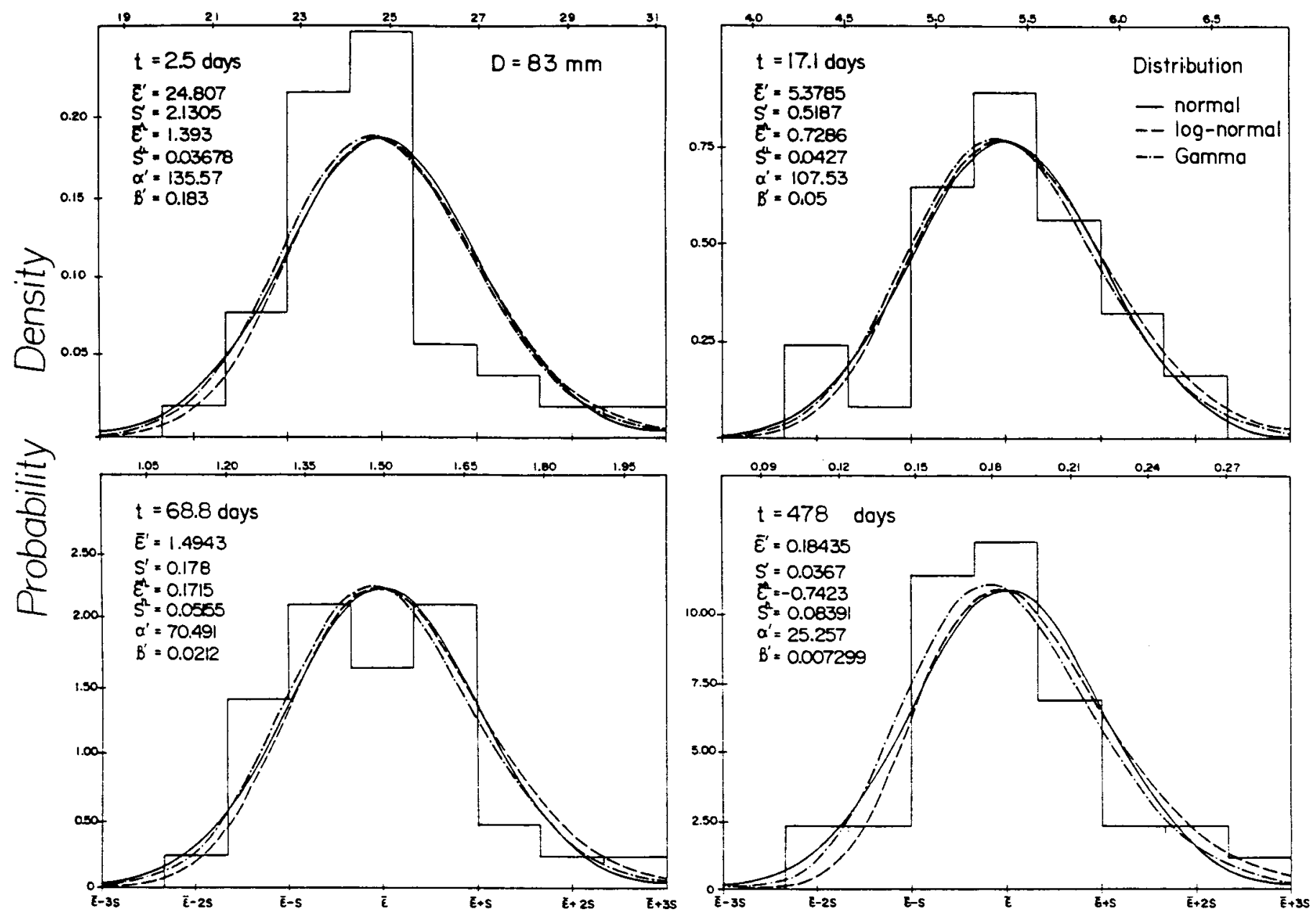

Shrinkage Strain Rate, $\dot{\varepsilon}_{s h}=\dot{\varepsilon}_{i, r}\left(\times 10^{6}\right)$

FIG. 12-Probability density distributions of shrinkage strain rate (Eq 2) for small diameter specimens. 

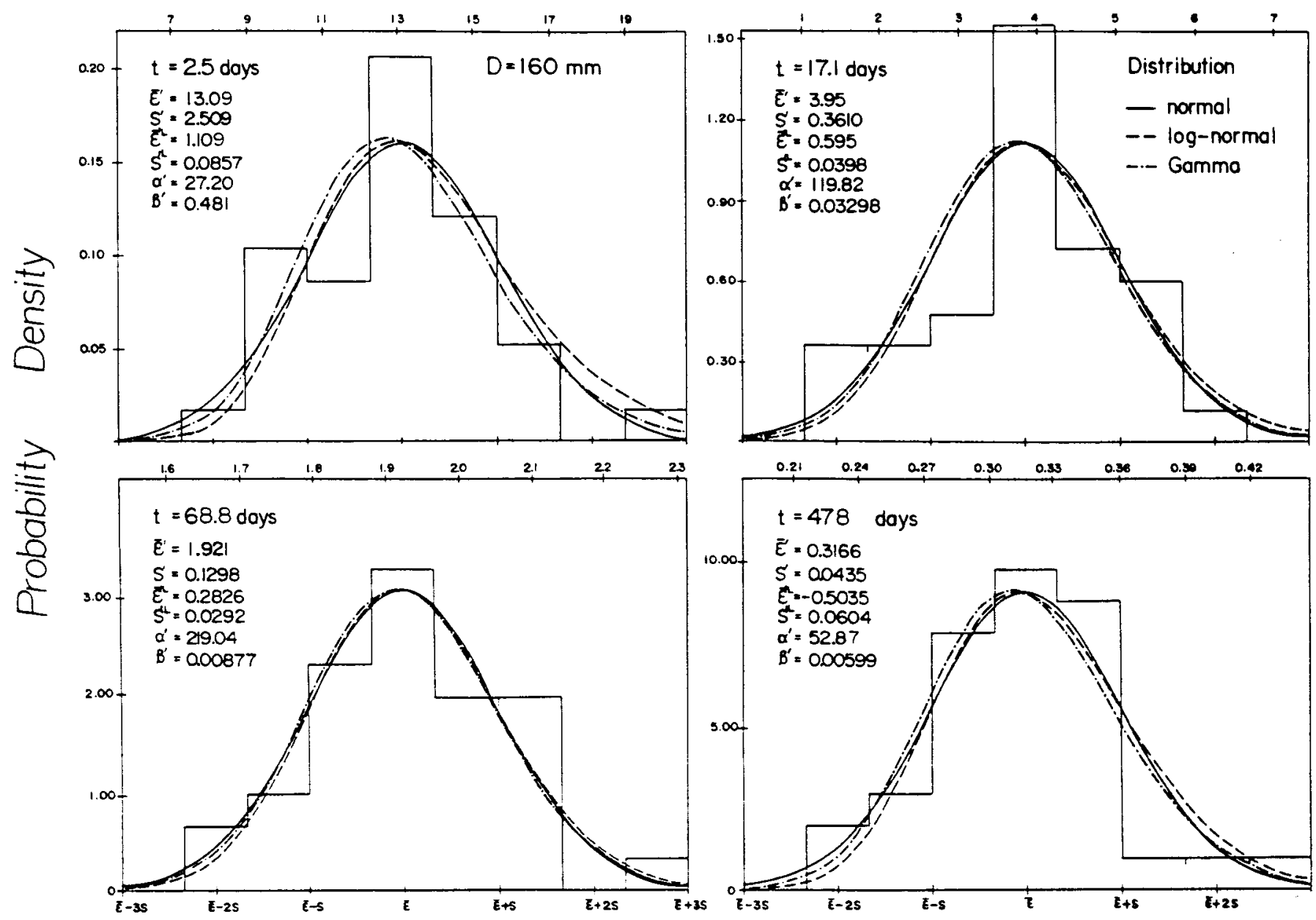

Shrinkage Strain Rate, $\dot{\varepsilon}_{\mathrm{sh}}=\dot{\varepsilon}_{i, r}\left(\times 10^{6}\right)$

FIG. 13-Probability densirv distributions of shrinkage strain rate for large diamer- 


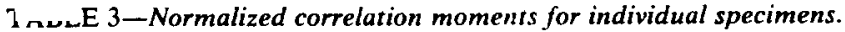

\begin{tabular}{|c|c|c|c|c|c|c|c|c|}
\hline \multicolumn{4}{|c|}{ Shrinkage Strain } & \multicolumn{5}{|c|}{ Shrinkage Strain Rate } \\
\hline$t_{r}$ & $t_{q}$ & $c_{r . q}$ & $c_{r . q}^{L}$ & $t_{r}$ & $t_{q}$ & $c_{r, q}^{\prime}$ & $c_{r . q}^{\prime L}$ & $c_{r .4}^{\log }$ \\
\hline \multicolumn{9}{|c|}{$\mathrm{D}=83 \mathrm{Mm}$} \\
\hline 3 & 3 & 1.000 & 1.000 & 2.5 & 2.5 & 1.000 & 1.000 & 1.000 \\
\hline 3 & 21 & 0.771 & 0.776 & 2.5 & 17.1 & 0.542 & 0.627 & 0.542 \\
\hline 3 & 91 & 0.718 & 0.725 & 2.5 & 68.8 & 0.196 & -0.153 & 0.201 \\
\hline 3 & 554 & 0.679 & 0.689 & 2.5 & 477.8 & 0.535 & -0.050 & 0.557 \\
\hline 3 & 1399 & 0.671 & 0.681 & 2.5 & 880.4 & 0.207 & 0.337 & 0.190 \\
\hline 21 & 21 & 1.000 & 1.000 & 17.1 & 17.1 & 1.000 & 1.000 & 1.000 \\
\hline 21 & 91 & 0.959 & 0.961 & 17.1 & 68.8 & 0.190 & -0.226 & 0.174 \\
\hline 21 & 554 & 0.935 & 0.939 & 17.1 & 477.8 & 0.487 & -0.065 & 0.530 \\
\hline 21 & 1399 & 0.942 & 0.946 & 17.1 & 880.4 & 0.342 & 0.329 & 0.369 \\
\hline 91 & 91 & 1.000 & 1.000 & 68.8 & 68.8 & 1.000 & 1.000 & 1.000 \\
\hline 91 & 554 & 0.985 & 0.986 & 68.8 & 477.8 & 0.317 & -0.191 & 0.318 \\
\hline 91 & 1399 & 0.983 & 0.985 & 68.8 & 880.4 & -0.472 & -0.468 & -0.457 \\
\hline 554 & 554 & 1.000 & 1.000 & 477.8 & 477.8 & 1.000 & 1.000 & 1.000 \\
\hline 554 & 1399 & 0.998 & 0.998 & 477.8 & 880.4 & 0.051 & -0.068 & 0.040 \\
\hline 1399 & 1399 & 1.000 & 1.000 & 880.4 & 880.4 & 1.000 & 1.000 & 1.000 \\
\hline \multicolumn{9}{|c|}{$\mathrm{D}=160 \mathrm{MM}$} \\
\hline 3 & 3 & 1.000 & 1.000 & 2.5 & 2.5 & 1.000 & 1.000 & 1.000 \\
\hline 3 & 21 & 0.895 & 0.889 & 2.5 & 17.1 & 0.276 & 0.423 & 0.234 \\
\hline 3 & 91 & 0.746 & 0.741 & 2.5 & 68.8 & 0.127 & 0.448 & 0.081 \\
\hline 3 & 554 & 0.561 & 0.554 & 2.5 & 477.8 & 0.558 & 0.537 & 0.565 \\
\hline 3 & 1399 & 0.561 & 0.554 & 2.5 & 880.4 & 0.426 & 0.213 & 0.409 \\
\hline 21 & 21 & 1.000 & 1.000 & 17.1 & 17.1 & 1.000 & 1.000 & 1.000 \\
\hline 21 & 91 & 0.950 & 0.950 & 17.1 & 68.8 & 0.656 & 0.592 & 0.656 \\
\hline 21 & 554 & 0.842 & 0.843 & 17.1 & 477.8 & 0.671 & 0.395 & 0.647 \\
\hline 21 & 1399 & 0.837 & 0.837 & 17.1 & 880.4 & 0.313 & -0.003 & 0.324 \\
\hline 91 & .91 & 1.000 & 1.000 & 68.8 & 68.8 & 1.000 & 1.000 & 1.000 \\
\hline 91 & 554 & 0.950 & 0.948 & 68.8 & 477.8 & 0.235 & -0.122 & 0.228 \\
\hline 91 & 1399 & 0.941 & 0.939 & 68.8 & 880.4 & 0.311 & 0.084 & 0.331 \\
\hline 554 & 554 & 1.000 & 1.000 & 477.8 & 477.8 & 1.000 & 1.000 & 1.000 \\
\hline 554 & 1399 & 0.994 & 0.994 & 477.8 & 880.4 & 0.328 & 0.158 & 0.348 \\
\hline 1399 & 1399 & 1.000 & 1.000 & 880.4 & 880.4 & 1.000 & 1.000 & 1.000 \\
\hline
\end{tabular}




$$
j J
$$




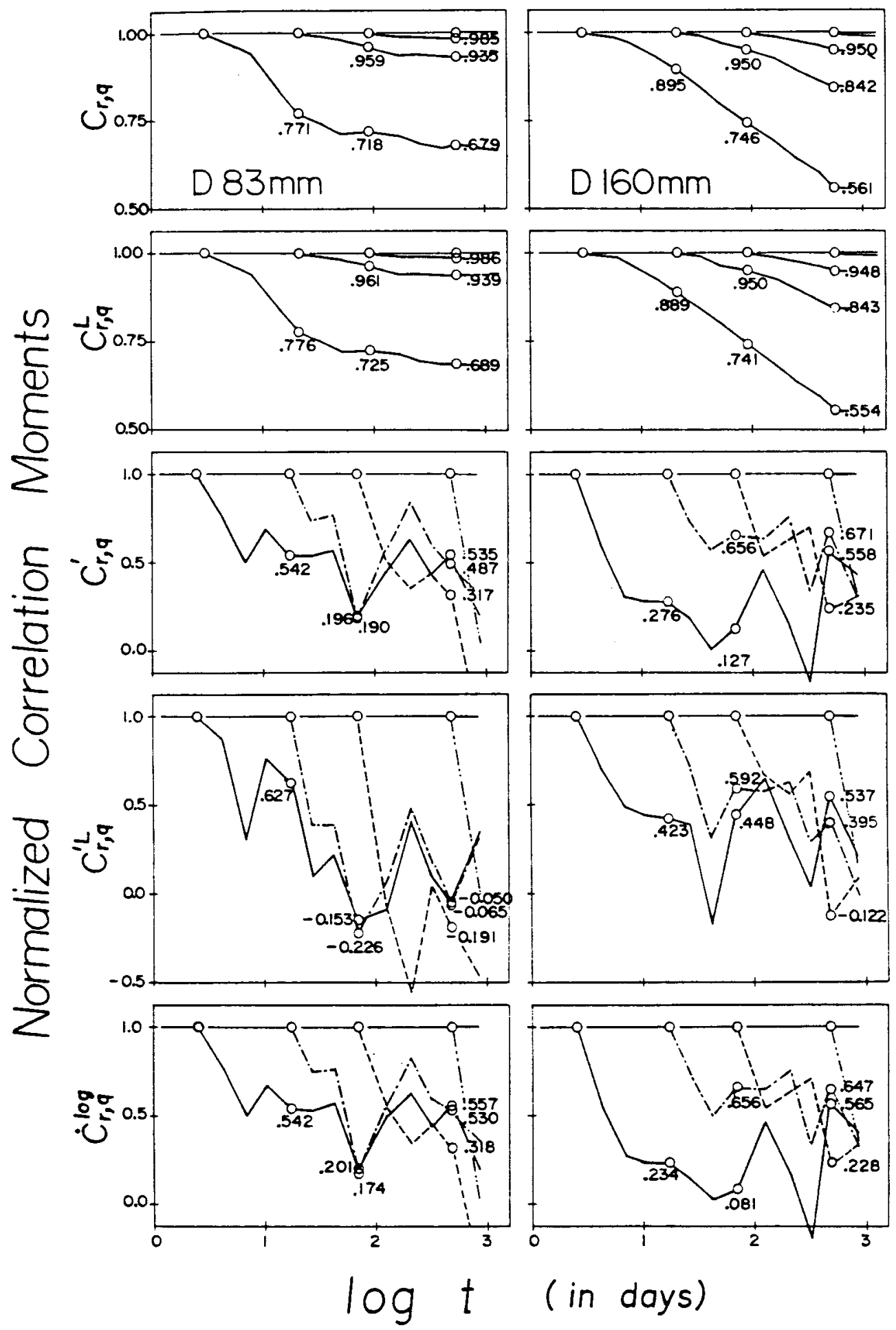

FIG. 15-Correlation between shrinkage values at various times. 

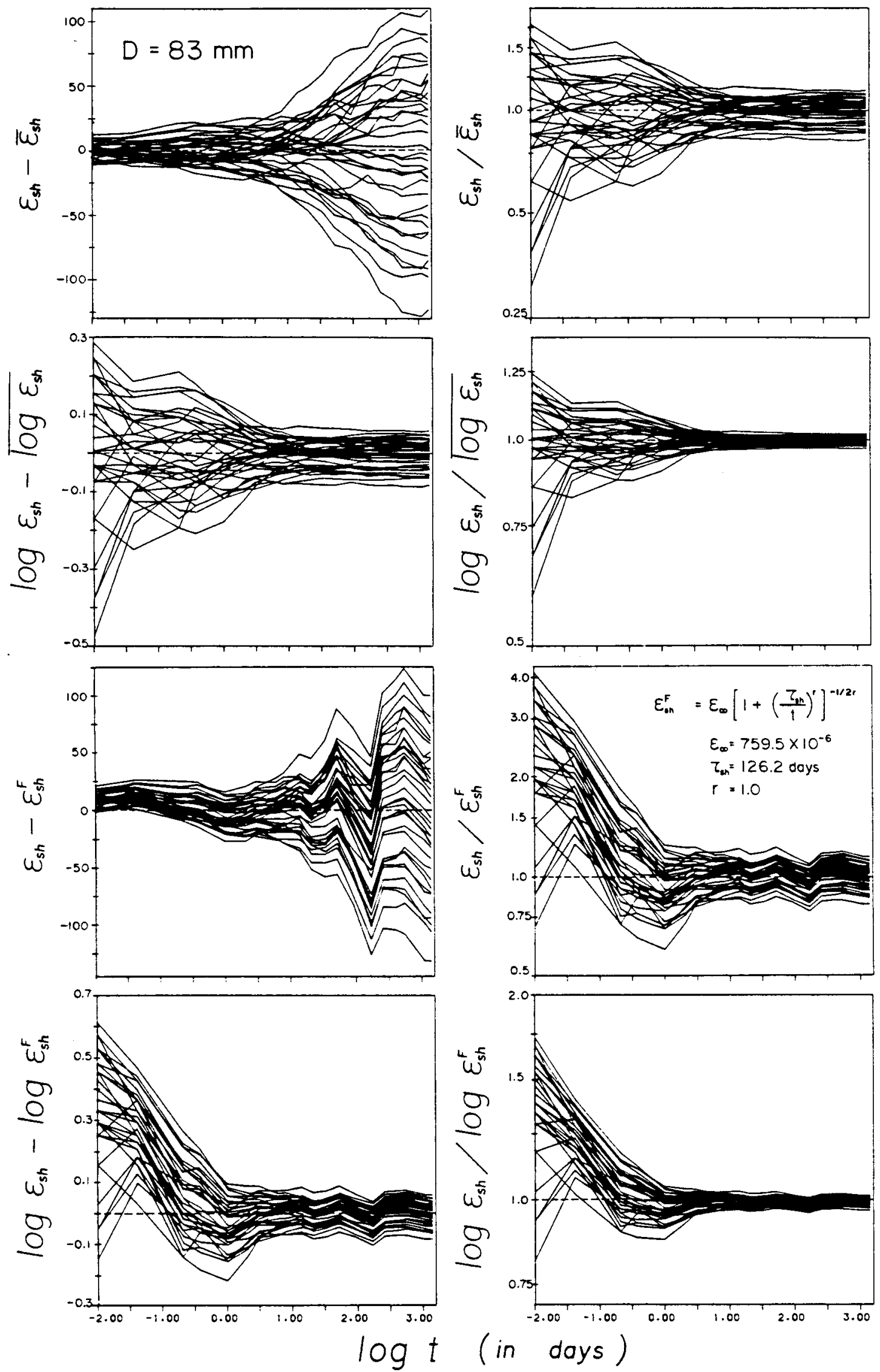

FIG. 16-For small diameter specimens, evolution of single-specimen deviation from the group mean for shrinkage strain and log-strain (top) and deviation of shrinkage strain from the optimum fit by formula (bottom). 

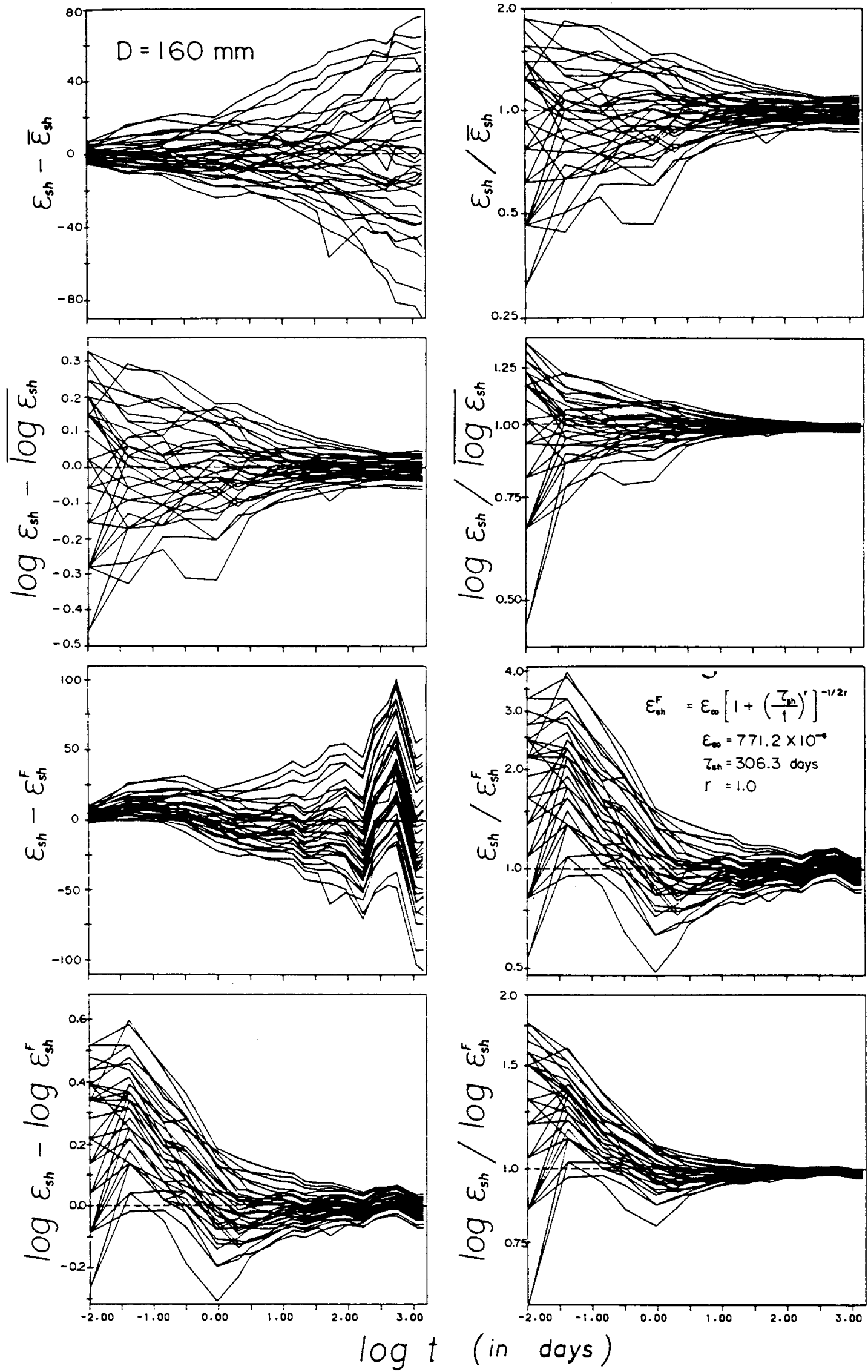

FIG. 17-For, large diameter specimens, evolution of single-specimen deviation from the group mean for shrinkage strain and log-strain (top) and deviation of shrinkage strain from the optimum fit by formula (bottom). 


\section{References}

[I] Tsubaki, T. et al., "Probabilistic Models," Chapter 5 of State-of-Art Report by RILEM Committee TC69 chaired by Z. P. Bažant, Preprints, Fourth RILEM International Symposium on Creep and Shrinkage of Concrete: Mathematical Modeling, Z. P. Bažant, Ed., Northwestern University, Evanston, IL, Aug. 1986, pp. 383-454 (preprint volume available from Northwestern University).

[2] Madsen, H. O. and Bažant, Z. P., "Uncertainty Analysis of Creep and Shrinkage Effects in Concrete Structures," ACI Journal, Vol. 80, Mar.-Apr. 1983, pp. 116-127.

[3] Bažant, Z. P. and Liu, K. L., "Random Creep and Shrinkage in Structures," Journal of Structural Engineering, ASCE, Vol. 111, 1985, pp. 113-134.

[4] Bažant, Z. P. and Panula, L., "Practical Prediction of Time-Dependent Deformations of Concrete," Materials and Structures, Parts I and II: Vol. 11, No. 65, 1978, pp. 307-328; Parts III and IV: Vol. 11, No. 66, 1978, pp. 415-434; Parts V and VI: Vol. 12, No. 69, 1979, pp. 169-183.

[5] Bažant, Z. P. and Panula, L., "Creep and Shrinkage Characterization for Analyzing Prestressed Concrete Structures," Prestressed Concrete Institute. Journal. Vol. 25, No. 3, 1980, pp. 86-122.

[6] Bažant, Z. P. and Panula, L., "New Models for Practical Prediction of Creep and Shrinkage," in Designing for Creep and Shrinkage in Concrete Structures, (Proceedings of A. Pauw Symposium held in Houston, 1978), ACI Special Publication SP.76, American Concrete Institute, Detroit, 1982, pp. 7-23.

[7] Alou, F. and Wittmann, F. H., "Etude expérimentale de la variabilité du retrait du béton," Proceedings, International Symposium on Fundamental Research on Creep and Shrinkage of Concrete. Lausanne, Sept. 1980, F. H. Wittmann, Ed., Martinus Nijhoff, Netherlands 1982, pp. 75-92.

[8] Reinhardt, H. W., Pat, M. G. M., and Wittmann, F. H., "Variability of Creep and Shrinkage of Concrete," Proceedings. International Symposium on Fundamental Research on Creep and Shrinkage of
Concrete, Lausanne, Sept. 1980, F. H. Wittmann, Ed., Martinus Nijhoff, Netheriands, 1982, pp. 95-108.

[9] Cornelissen. H., "Creep of Concrete-A Stochastic Quantity," Ph.D. thesis, Technical University, Eindhoven, The Netherlands, 1979 (in Dutch, with extended English summary).

[10] Cornelissen, H., "Creep of Concrete-A Stochastic Quantity," Proceedings. International Symposium on Fundamental Research on Creep and Shrinkage of Concretes, Lausanne, Sept. 1980, Martinus Nijhoff, Netherlands 1982, pp. 109-124.

[11] Bažant, Z. P., "Statistics of Shrinkage Test Data: Formulation and Method," Internal Report, Cooperative Project NU-EPFL, Northwestern University, Evanston, IL, May 1985.

[12] Bažant, Z. P., Wittmann, F. H., Kim, J.-K., and Alou, F., "Statistical Extrapolation of Shrinkage Data-Part I: Regression," ACI Ma. terials Journal, Vol. 34, Jan.-Feb. 1987, pp. 20-34.

[13] Bažant, Z. P., Kim, J.-K., Wittmann, F. H., and Alou, F., "Statistical Extrapolation of Shrinkage Data-Part II: Bayesian Updating," ACI Materials Journal, Vol. 84, Mar.-Apr. 1987, pp. 83-91.

[14] Bulmer, M. G., Principles of Statistics, Dover Publications, New York, 1979.

[15] Benjamin, J. R. and Cornell, A. C., Probability, Statistics and Decision for Civil Engineers, McGraw-Hill, New York, 1970.

[16] Blom, G., "Statistical Estimates and Transformed Data Variables," Ch. 12, J. Wiley, New York, 1958.

[17] Cinlar, E., Bažant, Z. P., and Osman, E., "Stochastic Process for Extrapolating Concrete Creep," Journal of the Engineering Mechanics Division, Proceedings of the American Society of Civil Engineers, New York, Vol. 103, 1977, pp. 1069-1088; Discussion 1979, pp. 485-489.

[18] Miller, I. and Freund, J., Probability and Statistics for Engineers, Prentice Hall, Englewood Cliffs, NJ, 1965, pp. 78-80.

[19] Kimball, B. F., "On the Choice of Plotting Positions on Probability Paper," Journal of the American Statistical Association. Vol. 55, No. 291, Sept. 1960, pp. 546-560.

[20] Martz, H. F. and Waller, R. A., Bayesian Reliability Analysis, J. Wiley, New York, 1982, p. 115. 